\title{
PARTIDOS Y LÍDERES EN LAS ELECCIONES GENERALES DE 2016 Y 2019. UNA VISIÓN EMOCIONAL
}

\section{Parties and leaders in the general elections of 2016 and 2019. An emotional vision}

\author{
MARÍA PEREIRA \\ Universidad de Santiago de Compostela \\ maria.pereira.lopez@usc.es \\ NIEVES LAGARES \\ Universidad de Santiago de Compostela \\ mnieves.lagares@usc.es \\ PAULO CARLOS LÓPEZ-LÓPEZ \\ Universidad de Santiago de Compostela \\ paulocarlos.lopez@usc.es \\ Cómo citar/Citation \\ Pereira, M. ${ }^{a}$, Lagares, N. y López-López, P. C. (2021). \\ Partidos y líderes en las elecciones generales de \\ 2016 y 2019 . Una visión emocional. \\ Revista de Estudios Políticos, 193, 211-249. \\ doi: https://doi.org/10.18042/cepc/rep.193.07
}

\section{Resumen}

Hace ya casi treinta años que ha comenzado el denominado giro afectivo en el ámbito de las ciencias sociales y con él han aparecido los primeros trabajos que profundizan en la importancia de la inclusión del componente emocional para el análisis del comportamiento, y en el caso que nos ocupa del comportamiento político. Partiendo de una revisión de estas aportaciones desde los ańos noventa del siglo xx en adelante, este trabajo pretende aportar evidencia empírica respecto de la importancia de la consideración de las emociones expresadas hacia los partidos políticos y los líderes a través de una visión basada en tres dimensiones de la emoción: presencia, intensidad y persistencia. Para llevar a cabo el análisis se utilizarán dos estudios demoscópicos propios realizados tras las elecciones generales de 2016 y 2019 en España. 


\title{
Palabras clave
}

Giro afectivo; emociones; política; partidos políticos; líderes; España.

\begin{abstract}
Almost thirty years ago there has been the so-called affective turn in the field of social sciences and with it the first studies begin to appear that deepen the importance of the analysis of the emotional component for the analysis of behavior, and in the case that we are concerned with political behavior. Based on a review of these contributions from the nineties of the twentieth century onwards, this study aims to provide empirical evidence regarding the importance of the consideration of emotions expressed towards political parties and leaders through a vision based on three dimensions of emotion: presence, intensity and persistence. Two own demoscopic studies carried out after the 2016 and 2019 General elections in Spain will be used to carry out the analysis.
\end{abstract}

\section{Keywords}

Affective turn; emotions; policy; political parties; leaders; Spain. 


\section{SUMARIO}

I. INTRODUCCIÓN. II. EMOCIONES Y POLÍTICA. EL GIRO AFECTIVO. III. OBJETIVOS Y METODOLOGÍA. IV. ANÁLISIS DE RESULTADOS. EMOCIONES, PARTIDOS Y LÍDERES EN ESPAÑA, 2016-2019: 1. Presencia emocional. 2. Intensidad emocional. 3. Persistencia emocional. 4. Construcción de componentes emocionales. V. CONCLUSIONES. BIBLIOGRAFÍA.

\section{INTRODUCCIÓN}

Durante años, las explicaciones en la ciencia política estuvieron marcadas por una cierta hegemonía de las teorías de la elección racional, que hacía girar la explicación sobre la simple y mera racionalidad del individuo basada en un supuesto cálculo coste-beneficio. La elección racional constituye el éxtasis del predominio racionalista en las ciencias sociales, y si bien los modelos surgidos al amparo del enfoque hiperracional se hicieron cada vez más complejos, tratando de incorporar dimensiones complementarias de la propia racionalidad, lo cierto es que siempre tuvieron un elemento vehicular común: la preminencia de la razón como eje de la explicación de los comportamientos y, en consecuencia, el desprecio o menosprecio, de lo emocional-afectivo como elemento explicativo de las percepciones hacia la política y del comportamiento político de los individuos.

Esto comenzaría a cambiar hace unos treinta años, cuando desde diferentes sectores tanto internos como externos ${ }^{1}$ a las ciencias sociales se empieza a reivindicar una visión menos reduccionista de la explicación política, más inclusiva, que rompe con el encorsetado e inmovilista legado que había impuesto la teoría de la elección racional. Esta ruptura, conocida ya como el denominado "giro afectivo" (Nussbaum, 1994; Hogget y Thompson, 2012; Arias Maldonado, 2016), vendría a reivindicar el papel de los afectos, las emociones, los sentimientos y las pasiones como elementos consustanciales a la comprensión de la política, así como de la percepción que los individuos tienen de esta. La nueva apuesta abriría un espacio infinitamente rico desde el punto de vista científico, tanto inter como multidisciplinar, en el

1 A la hora de explicar este cambio es importante señalar el peso de los estudios dentro de la neurociencia y la neurofisiología, así como de los avances tecnológicos en las mismas. 
que nos encontramos con importantes aportaciones desde los más diversos campos, que afrontaron un terreno hasta el momento poco explorado, bien fuera por imposiciones paradigmáticas, por desconocimiento o por la comodidad que da el saberse dentro de un terreno asentado y de escaso riesgo.

La incisión que el componente emocional tiene en la política comienza por romper con la tradicional dicotomía razón/emoción, apostando al mismo tiempo no por un dualismo excluyente, sino por una "simbiosis explicativa", en la que razón y emoción son elementos complementarios que no antagónicos (Máiz, 2010, 2011) que enriquecen y facilitan la explicación política. Esta apuesta será la que guíe un importantísimo número de trabajos científicos, bien de marcado carácter teórico-normativo (Bodei, 2003; Solomon, 1993; Damasio, 1994, 2003; Elster 1999a, 1999b; Nussbaum 1994, 2001, 2013), bien de carácter empírico (Ciuk et al., 2015; Neuman et al., 2007; Marcus et al. 2000, 2005, 2006, 2017; Watson, 1997; Watson y Tellegen, 1999; Bradley y Lang, 1994; Marcus y Mckuen, 1995; Russell, 1980; Abelson et al., 1982), que comparten una única meta, reivindicar el papel de las emociones como un elemento explicativo central, así como una cuestión fundamental que durante mucho tiempo impidió o al menos sirvió de excusa para retrasar su estudio, la capacidad de medir las emociones, y en consecuencia, de comprenderlas. Así, las emociones dejaban de ser un elemento escurridizo, complejo y no aprehensible, al tiempo que se dotaban de los fundamentos teóricos y empíricos que facilitaban y abrían un importante campo de estudio en torno a ellas.

En el trabajo que se presenta en estas páginas nos gustaría revisar cuáles han sido los ejes centrales que están detrás de por qué surge y sobre qué se sustenta el denominado giro afectivo en las ciencias sociales y cuál es la evolución y estado actual de las investigaciones que dentro de la ciencia política se han centrado en el estudio de las emociones; para terminar exponiendo algunos de los principales hallazgos de nuestra investigación, obtenidos a través de estudios demoscópicos propios, respecto a la presencia, intensidad y persistencia de las emociones expresadas por la ciudadanía hacia los partidos políticos españoles y los líderes de estos en los últimos cinco años.

\section{EMOCIONES Y POLÍTICA. EL GIRO AFECTIVO}

Quizás hoy ya no podríamos entender la política sin el componente emocional que lleva aparejado (Redlawsk, 2006) o, más bien, siempre hemos sabido de la existencia e importancia de dicho componente en la explicación de nuestra relación como individuos con ella, pero hoy somos realmente 
conscientes de su valor y lo que es más importante, empezamos a tener herramientas que nos permiten estudiarlas y analizarlas. Y esto último es, posiblemente, la cuestión fundamental que permite explicar el porqué del sinfin de trabajos que en las últimas tres décadas han surgido en torno a esta cuestión, así como las diferentes aproximaciones que nos podemos encontrar en este camino.

Pero la importancia que la capacidad de aprehender las emociones ha tenido para las ciencias sociales en general y para la ciencia política en particular, no se sustenta sobre arenas movedizas, sino todo lo contrario; son fundamentales las aportaciones que desde la neurociencia, la psicología, la filosofía o la teoría política comenzaron a surgir a principios de la década de los noventa reivindicando, como mencionábamos en la introducción de este artículo, un nuevo estatus para las emociones frente a la lógica racional que había dominado el panorama científico-social hasta el momento. Algunos de estos trabajos se remontan hasta la filosofía de Spinoza ${ }^{2}$ como base para abordar la importancia y naturaleza de los afectos humanos, afirmando, como señala Damasio (1994, 2003), que habría sido aquel el primero en adivinar el error cometido por Descartes y, en consecuencia, la importancia de la relación existente entre cuerpo y mente ${ }^{3}$ y todo lo que ello conlleva en términos de comprensión de la acción humana. Una visión que en cierta medida comparte Bodei (2003), quien entiende que en la obra de Spinoza está la clave para entender, desde un punto de vista político, el conflicto existente durante siglos entre la razón y las pasiones.

Pero si hay una cuestión más allá de la relación entre razón y emoción que haya preocupado y, por tanto, estado presente en estos primeros trabajos, es la definición de las emociones y su diferenciación respecto a sentimientos, afectos y pasiones. Son los casos de Solomon (1993), quien dibuja una teoría subjetiva de las pasiones, profundizando en cuál es la lógica que se encuentra detrás de la emoción; el propio Damasio (1994), quien las distingue de los sentimientos y las define como un conjunto de respuestas químicas y neuronales que conformarían un patrón distintivo, una suerte de «huella emocional»

2 Baruch Spinoza abordó a lo largo de su vida, la naturaleza de emociones tales como el amor o el deseo, reivindicando, a pesar de los límites que les impondrá, su importancia y renegando, a su vez, de quienes «detestaban y ridiculizaban los afectos», abogando al mismo tiempo por la necesidad de intentar comprenderlos y entendiendo que es posible seguir un orden respecto a ellos.

3 Esta distinción es fundamental, pues como señala Damasio, Spinoza habría allanado el terreno sobre el que siglos más tarde se asentaría la moderna neurofisiología y, en consecuencia, la posibilidad de conocer en detalle el funcionamiento del cerebro y la naturaleza de emociones y sentimientos. 
que intervendría en nuestro proceso de toma de decisiones ${ }^{4}$, o Dixon (2003), quizás uno de los autores que en mayor medida se ha esforzado por intentar diferenciar semánticamente entre emociones, sentimientos, afectos y pasiones.

Otro elemento presente en estos primeros trabajos y en otros muchos que han venido después es la implicación de las emociones en la definición del comportamiento humano, y en consecuencia, también en el comportamiento político. En este sentido, si bien Solomon (1993) ya apuntaba algunas cuestiones, son fundamentales las aportaciones de Nussbaum, quien profundiza en el rol que las emociones juegan sobre nuestro pensamiento (1994, 2001), o de Elster (1999a, 1999b), quien para comprender la construcción del comportamiento humano aborda los procesos de cognición, elección y racionalidad y cómo estos se ven socavados por los procesos físicos subyacentes a las emociones. Años después, será Redlawsk (2006) quien explorará las relaciones existentes entre afecto y cognición, así como las implicaciones que ambos tienen para el proceso de evaluación, decisión y acción política.

Avanzando en una visión más aplicada en lo que al estudio de las emociones se refiere, dos son las cuestiones fundamentales que han construido el debate: la acotación de tipos de emociones y la delimitación de las herramientas y técnicas que puedan permitir su análisis. Respecto a lo primero, es imposible no mencionar la gráfica aportación de Plutchik, (2002) en su rueda de las emociones, donde se aborda la posibilidad de diferenciar entre emociones básicas y emociones compuestas, así como la definición de estas últimas como el resultado de la combinación de las primeras. En esta misma línea los trabajos de Marcus $(2000,2002)$ y Marcus et al. (2000, 2005), quienes partiendo de su teoría de la inteligencia afectiva profundizan en la activación cognitiva que se encontraría detrás de la presencia de las diferentes emociones que experimentamos como individuos ante diversas situaciones. Estos últimos trabajos entroncarían a su vez con otras aportaciones, que responderían a la segunda de las cuestiones planteadas: el diseño de herramientas y técnicas que permitan delimitar qué emociones analizar y cómo hacerlo (Conover y Feldman, 1986; Bradley y Lang, 1994; Marcus et al. 2005, 2006, 2017; Ciuk et al., 2015). En todos ellos encontramos, bien recurriendo a técnicas experimentales (Bradley y Lang, 1994; Ciuk et al., 2015; Marcus et al., 2017), bien a técnicas cuantitativas como los estudios demoscópicos (Marcus y Mackuen, 1993; Marcus et al., 2000), consistentes intentos por encontrar la forma más robusta de aproximarse a emociones concretas, o bien de establecer baterías y/o constructos emocionales a través

4 Damasio entiende que las emociones preceden a los sentimientos, pero que ambos son una guía para la toma de decisiones. 
de diferentes análisis estadísticos más o menos sofisticados (correlaciones, análisis factorial confirmatorio o modelos de ecuaciones estructurales).

Estos trabajos han sido fundamentales para la evolución de los estudios dentro de la ciencia política, pues si muchos de ellos han partido de la psicología cognitiva, bien por formación de sus autores, o bien por los enfoques teóricos de los que parten para la elaboración de sus planteamientos, lo cierto es que gran parte de ellos, como es el caso de los trabajos en solitario o en compañía de Marcus, han aplicado su metodología al estudio de la política. Así, en los últimos años nos encontramos con un buen número de estudios que han analizado la importancia de las emociones y su relación con diferentes aspectos de la política. En este sentido podemos destacar los trabajos en torno a la construcción de la emoción colectiva y el papel de la identidad en la política (Hogget, 2010) e incluso en la construcción de la vida política en su conjunto (Westen, 2007), la influencia que emociones negativas como miedo o enfado tienen sobre la aparición de actitudes populistas (Rico et al., 2017), el efecto que dichas emociones tienen en el apoyo electoral a los partidos de extrema derecha (Vasilopoulos et al., 2019; Marcus et al., 2019), la importancia del factor emocional en la percepción de los líderes políticos y sus actuaciones (Abelson et al., 1982, Masters y Sullivan, 1989, Sullivan y Masters, 1988, Ragsdale, 1991, Masters, 2001), la delimitación de las dimensiones emocionales específicas de cada campaña electoral que influirían en las evaluaciones de los votantes (Yates, 2016), el peso de los componentes emocionales en la construcción de voto a los partidos (Jaráiz et al., 2020a) o incluso la importancia emocional que se encuentra detrás de la movilización política de protesta (Jasper, 2012). También notorios han sido los trabajos que se han realizado en el ámbito de la comunicación política, y más concretamente en el efecto emocional de las redes sociales (Toubiana y Zietsma, 2017) y de los mensajes transmitidos por los partidos y sus candidatos a través de ellas (Stieglitz y Dang-Xuan, 2012, Lin y Utz, 2015, Hasell y Weeks, 2016, Brady et al., 2017, López-López et al., 2020, Jaráiz et al., 2020b, 2020c).

Dos elementos se encuentran detrás de la mayoría de los trabajos que hemos mencionado: en primer lugar, la mencionada ruptura de la dicotomía razón y emoción, y la consideración de esta como un elemento fundamental para el análisis político; y segundo, la construcción social de las emociones y, ligada a ella, la importancia del proceso de aprendizaje cognitivo que la vincula.

\section{OBJETIVOS Y METODOLOGÍA}

El objetivo principal de este artículo es contribuir a la línea de trabajo que ha abordado la relación entre las emociones y la política descrita en líneas precedentes, y más concretamente, al análisis sobre la percepción que la 
ciudadanía tiene de las emociones que los partidos políticos y sus líderes les generan. En las próximas páginas intentaremos ilustrar algunas de estas cuestiones, procediendo con un análisis descriptivo y multivariante sobre cómo han cambiado las emociones expresadas por los ciudadanos españoles hacia los partidos políticos y sus líderes entre 2016 y 2019. Algunas de las hipótesis de trabajo que surgen de las preguntas de investigación que nos planteamos en la introducción de este trabajo son: a) hay una diferencial emocional que los ciudadanos sienten/muestran hacia los partidos políticos y sus líderes entre ambos procesos electorales, especialmente en términos de intensidad; b) hay una mayor presencia de emociones negativas y muy negativas que de emociones positivas hacia líderes y partidos en ambos procesos; c) los niveles de presencia e intensidad emocional hacia líderes y partidos muestran valores muy similares en ambos procesos; d) las emociones expresadas hacia líderes y partidos políticos pueden ser agrupadas en componentes emocionales positivos, negativos y muy negativos, con similares niveles de compactación interna.

Para llevar a cabo esta tarea, utilizaremos las bases de datos de los estudios demoscópicos realizados por el Equipo de Investigaciones Políticas de la USC (EIP-USC) tras las elecciones generales de 2016 y de noviembre de 2019 en España (tabla 1). Antes de exponer los principales hallazgos obtenidos en nuestro trabajo, nos gustaría explicar brevemente la evolución de la investigación que representan estos estudios, pues entendemos que es fundamental comprender el diseño analítico-metodológico que se encuentra detrás, para comprender con claridad los resultados que se presentarán a continuación.

Tabla 1. Estudios demoscópicos sobre emociones en España (EIP-USC)

\begin{tabular}{|c|c|c|}
\hline Ańo & Estudio & Características técnicas \\
\hline 2017 & $\begin{array}{l}\text { Estudio politico } \\
\text { elecciones generales } \\
\text { en España } 2016 . \\
\text { Emociones y } \\
\text { comportamiento } \\
\text { electoral (EPEGE. } \\
\text { Emociones y } \\
\text { comportamiento } \\
\text { electoral 2016) }\end{array}$ & $\begin{array}{l}\text { Fechas: } 17 \text { de mayo- } 21 \text { de junio de } 2017 . \\
\text { Universo: población mayor de } 18 \text { años, } \\
\text { residente en Espańa. } \\
\text { Tamaño muestral: } 1000 \text {. } \\
\text { Supuestos: } p=q \text {. } \\
\text { Error asociado: } \pm 3,16 \% \text {. } \\
\text { Afijación: proporcional (cuotas de sexo y edad). } \\
\text { Administración: telefónicamente mediante el } \\
\text { sistema CATI. }\end{array}$ \\
\hline
\end{tabular}

5 Por el momento en el que fue realizado y en un intento de evitar posibles problemas en el trabajo de campo, derivados de las negociaciones para la formación de Gobierno, 


\begin{tabular}{|c|c|c|}
\hline Año & Estudio & Características técnicas \\
\hline 2020 & $\begin{array}{l}\text { Estudio postelectoral } \\
\text { elecciones generales } \\
\text { en España noviembre } \\
2019 \text { (EPEGE } \\
\text { noviembre 19) }\end{array}$ & $\begin{array}{l}\text { Fechas: } 14 \text { de enero- } 22 \text { de marzo de } 2020 \text {. } \\
\text { Universo: población mayor de } 18 \text { años, } \\
\text { residente en Espańa. } \\
\text { Tamaño muestral: } 1000 \text {. } \\
\text { Supuestos: } p=q \text {. } \\
\text { Error asociado: } \pm 3,1 \% \text {. } \\
\text { Afijación: proporcional (cuotas de sexo y edad). } \\
\text { Administración: telefónicamente mediante el } \\
\text { sistema CATI. }\end{array}$ \\
\hline
\end{tabular}

Fuente: elaboración propia a partir de banco de datos del EIP-USC.

El interés en el análisis del comportamiento electoral llevó hace unos seis años a abordar nuevas líneas de trabajo que permitieran contribuir a explicar, en cierta medida, el declive de algunos de los elementos tradicionales en la explicación del voto y la aparición de otros nuevos. Así se introduce el estudio y análisis de las emociones como elemento de explicación del comportamiento político en general y del electoral en particular. El primer trabajo realizado fue el Estudio postelectoral elecciones generales 2015 (2016), en el cual se incluyó la percepción de los ciudadanos respecto de las emociones experimentadas hacia los partidos políticos y su liderazgo.

En esta primera medición se detectaron algunos problemas metodológicos (Jaráiz et al., 2020a) que motivaron la revisión de diversas investigaciones en este terreno (Marcus y Mackuen, 1993; Marcus et al., 2000, 2005, 2017) y que concluyeron en la realización de un nuevo estudio en el año 2017, Estudio político elecciones generales en España 2016. Emociones y comportamiento electoral ${ }^{6}$. Este planteamiento permitió obtener resultados más robustos a los

no se trata de un estudio postelectoral al uso, sino de un estudio de carácter político muy amplio, que aborda entre otras cuestiones referencias al proceso electoral de 2016 y al escenario político derivado del mismo.

6 Este nuevo planteamiento implicaba, fundamentalmente, introducir una pregunta de filtro de tipo nominal, con la que se concluía si el entrevistado/a había sentido o no en alguna ocasión una determinada emoción hacia un líder o partido. Si la respuesta era afirmativa, se le planteaba cuál era el grado de intensidad con la que la había experimentado y, por último, se daba cuenta de si seguía o no sintiendo dicha emoción (ver Jaráiz et al., 2020). Además, otro cambio importante fue la modificación del rango escalar de la pregunta de intensidad, pasando de 0 a 10 en el año 2016 a una escala 1-5 en el año 2017, lo que simplificaba notablemente las respuestas de los 
obtenidos en el primer estudio y reducir en gran medida el factor de no respuesta $^{7}$. La potencialidad del análisis de las emociones en este segundo trabajo fue notable y permitió la continuidad del esquema en estudios posteriores, tanto nacionales como autonómicos ${ }^{8}$ y municipales.

Entrando ya en cuestiones más concretas respecto al modelo analítico-metodológico adoptado, uno de los primeros y principales escollos fue la inexistencia de acuerdo en el ámbito de la psicología cognitiva, y mucho menos en el de la ciencia política, sobre cuáles son las emociones concretas que se deben medir para explicar el comportamiento político. Si bien las propuestas desde ambas áreas de conocimiento en las que se ha abordado la medición de las emociones son innumerables (Ciuk et al., 2015; Neuman et al., 2007, 2018; Marcus et al. 2000, 2006, 2017; Watson, 1997; Watson y Tellegen, 1999; Bradley y Lang, 1994; Marcus y Mckuen, 1993; Abelson et al., 1982), los trabajos en nuestro país eran inexistentes?. Por ello, tomando como referencia además de las propuestas citadas los estudios realizados por la ANES, nos decantamos por seguir el planteamiento propuesto por Marcus et al. $(2000)^{10}$, debido en parte a que había sido testado en varias ocasiones en el

entrevistados. Esta última decisión estuvo motivada principalmente por el hecho de seguir el patrón utilizado por la ANES en sus estudios, pionera en la medición de las emociones y base para la realización de algunos de los principales trabajos sobre emociones que han sido publicados y mencionados en este artículo.

7 La reducción del factor de no respuesta es un elemento fundamental, habida cuenta de los problemas que acarrea a posteriori en el análisis de datos, especialmente en lo relativo a su validez y fiabilidad.

8 Tras estos dos primeros trabajos en el ámbito nacional, se realizó el primer estudio a nivel autonómico, concretamente en Cataluńa, tras las elecciones autonómicas de 2017, Estudio postelectoral emociones en Cataluña. La situación catalana representaba una estructura de oportunidad evidente por la implicación que las emociones tenían y tienen en la estructuración del procès. Posteriormente en 2018, se realizaría un nuevo estudio autonómico tras las elecciones en Andalucía, Estudio postelectoral elecciones autonómicas en Andalucía 2018.

9 Más allá de algún estudio político realizado por el Centro de Investigaciones Sociológicas en el que se incluye una pregunta sobre los sentimientos expresados por los españoles hacia la política, y de algún estudio del Centre d'Estudis d'Opinió que recoge alguna pregunta sobre las emociones expresadas por los catalanes hacia determinados acontecimientos políticos del procés, no existen en nuestro país organismos que hayan realizado estudios específicos sobre las emociones hacia la política, o que al menos hayan dedicado un apartado específico a las mismas.

$10 \mathrm{Si}$ bien los autores referencian el estudio de las emociones que los individuos sienten hacia los líderes políticos, en nuestro estudio hemos incluido estas referencias no solo hacia los líderes, sino también hacia los partidos políticos. Entendemos que esta 
ámbito del comportamiento político (Conover y Feldman, 1986; Marcus, $2000,2006,2017)$ y planteaba, por otro lado, la ventaja ańadida de haber intentado agrupar las emociones individuales en componentes emocionales a través de modelizaciones estadísticas multivariantes. Si bien partimos desde un punto de vista empírico del enfoque metodológico de los trabajos ya mencionados «compartimos ciertas reservas con los autores respecto a la consideración, desde el ámbito teórico, del impacto del componente emocional [...], abogamos por una visión no antagónica de ambos componentes, racional y emocional, sino complementaria; ambos componentes no constituyen una dicotomía, sino que caminan juntos, de modo que la razón en política no existe independientemente de las emociones y a la inversa» (Jaráiz et al., 2020a: 118).

El esquema analítico-metodológico definitivo por el que nos decantamos para llevar a cabo la medición del componente emocional, tras el primer estudio realizado en 2016, parte de la formulación de tres preguntas concretas con las que se evalúan tres dimensiones de la emoción que el ciudadano expresa hacia dos objetos concretos: los partidos políticos y los líderes de estos (esquema 1). La primera dimensión analizada, y de la que surgen las dos siguientes, es la presencia de la emoción, es decir, si el individuo afirma haber sentido o sentir en el momento en el que se realiza la encuesta, una emoción concreta sobre un determinado líder y/o partido. Una vez realizada esta primera cuestión se mide la intensidad con la que se ha expresado dicha emoción, por lo que la pregunta se formula únicamente a aquellos individuos que contestaron afirmativamente a la primera cuestión. Y finalmente, con el objetivo de evaluar la persistencia emocional, se pregunta al individuo si dicha expresión se mantiene o no en el tiempo. Estas tres dimensiones nos dan una visión muy completa de cómo expresamos nuestras emociones en política, mejorando notablemente la formulación utilizada en el primer estudio.

Una vez definido este esquema se acotó la batería de emociones sobre las que se preguntaría. Como ya avanzamos, esta no era una decisión sencilla; así, frente al primer estudio de 2016, en el que se incluyeron un total de nueve emociones, en los estudios realizados a partir de 2017 se incluyeron un total de trece $^{11}$. Las emociones utilizadas pueden ser a su vez agrupadas en tres componentes emocionales, siguiendo el esquema de Marcus $(2000,2002)$ y

es una cuestión relativa al contexto del sistema político en el que se encuadra el trabajo de los autores, Estados Unidos, frente a nuestro caso, donde consideramos que la identificación con la marca de partido es fundamental.

11 A esta ampliación hacia un total de doce emociones analizadas, se decidió añadir una más, tranquilidad, teniendo en cuenta los resultados de presencia de esta emoción que había arrojado la explotación de los datos del trabajo realizado en 2016. 
ampliado posteriormente (Marcus et al., 2017) ${ }^{12}$, que abogan por la sustitución de la tradicional división dentro de la psicología entre dimensiones positivas y negativas de las emociones, por los términos entusiasmo para referirse a la dimensión o afecto positivo (entusiasmo, esperanza, orgullo), ansiedad para referirse a la dimensión o afecto negativo (ansiedad, enfado, miedo, preocupación) y aversión para referirse a la dimensión o afecto muy negativo (amargura, asco, desprecio, odio, resentimiento $)^{13}$.

\section{Esquema 1. Esquema analítico-metodológico del concepto emoción}

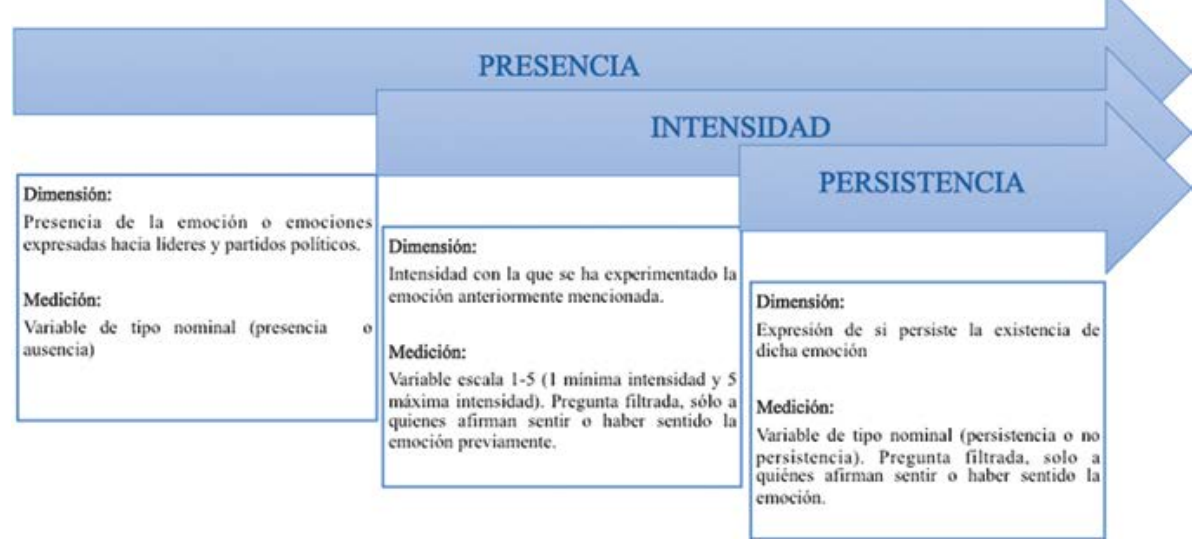

Fuente: elaboración propia a partir de banco de datos del EIP-USC.

Aclaradas estas cuestiones, a continuación y utilizando, como ya se ha indicado, los estudios demoscópicos realizados tras la celebración de las elecciones

12 Siguiendo los planteamientos definidos por los autores en su exposición de la teoría de la inteligencia afectiva, cada uno de estos componentes se correspondería con los sistemas cognitivos — sistema disposicional y sistema de vigilancia- que el cerebro activa en cada momento en función de la reacción que determinados hechos, acontecimientos o personas les hacen experimentar en su vida diaria a los individuos. Estos mecanismos cognitivo-emocionales los empleamos en cualquier ámbito o escenario de nuestra vida y, en consecuencia, también en nuestra interactuación con el sistema político.

13 Este componente, que inicialmente no contemplaban, sería el resultado de la descomposición de las nueve emociones que inicialmente componían el término ansiedad en dos componentes emocionales distintos, que implican a su vez dos sistemas cognitivos diferenciados. 
generales de 2016 y noviembre de 2019, llevaremos a cabo en los siguientes apartados una aproximación descriptiva y multivariante de la distribución que la presencia, intensidad y persistencia emocional hacia los principales partidos políticos y sus líderes expresaron los ciudadanos en estos momentos clave del sistema político español. La justificación de habernos decantado por ambos estudios recae en tres consideraciones de importancia: la primera tiene que ver con el interés por comparar dos casos que comparten una importante variable: el hecho de haber sido ambos procesos electorales repetidos, aunque sea por motivos diferentes; el segundo, que en contraposición al estudio de 2015, ambas encuestas utilizan el mismo esquema metodológico para la medición de las emociones, y en tercer lugar, la diferencia temporal entre ambos, la cual brinda la oportunidad de analizar de manera longitudinal la percepción emocional de los españoles a la vista de los cambios que se han producido en nuestro sistema político en este tiempo, en especial el asentamiento de los nuevos partidos y la aparición de la extrema derecha.

\section{ANÁLISIS DE RESULTADOS. EMOCIONES, PARTIDOS Y LÍDERES EN ESPAÑA, 2016-2019}

\section{PRESENCIA EMOCIONAL}

Comenzamos con el análisis descriptivo de la presencia emocional (en porcentaje) hacia los principales partidos políticos, comparando los valores en 2016 y 2019 (gráfico 1) ${ }^{14}$. En el caso del Partido Popular (PP), en ambos momentos las emociones que presentan mayores niveles de presencia son el enfado $(55,3 \%$ en $2016,26,6 \%$ en 2019$)$ y la preocupación $(54,6 \%$ en 2016, 30,2\% en 2019), dos emociones ligadas al factor ansiedad; por el contrario, la emoción que muestra una menor presencia es el odio $(9,1 \%$ en 2016, 2,4\% en 2019). Una cuestión importante teniendo en cuenta los cambios por los que ha atravesado la formación en estos años es el hecho de que, en líneas generales, los porcentajes de presencia son menores en el año 2019 para todas las emociones analizadas.

El panorama es un tanto distinto en lo que al Partido Socialista (PSOE) se refiere. Las dos emociones que presentan mayores niveles de presencia en

14 Hacemos constar que en los gráficos y tablas hemos incluido la emoción Asco, si bien en el ańo 2016 y como ya se avanzó, esta emoción se tradujo por Disgusto. Para simplificar aparece un único término, si bien se aclara al lector que para el año 2016, el término fue Disgusto y para el año 2019, Asco. 
ambos ańos son, por este orden, la esperanza (38,2\% en 2016, 41,1\% en 2019) y la preocupación ( $43,5 \%$ en $2016,34,6 \%$ en 2019), alternando primer y segundo puesto. Al igual que sucedía con el PP, la emoción que menor presencia muestra es el odio (2,9\% en $2016,1,5 \%$ en 2019$)$, seguida de cerca por el desprecio, ambas emociones pertenecientes al componente aversión. Un elemento de importancia en el caso del PSOE es el incremento en el año 2019 de la presencia de tranquilidad (19,1\% en 2016, 22,7\% en 2019), un patrón que solo se producirá, como veremos, en el caso de esta formación y de Podemos.

Preocupación $(43,4 \%)$ y miedo $(37 \%)$ son las dos emociones que en mayor medida expresaban los españoles hacia Podemos en el año 2016, un planteamiento que cambia de forma notable en 2019, siendo sustituida la primera por la esperanza $(38 \%)$ y la segunda por la preocupación $(32,9 \%)$.

Gráficos 1. Presencia emocional hacia partidos 2016-2019
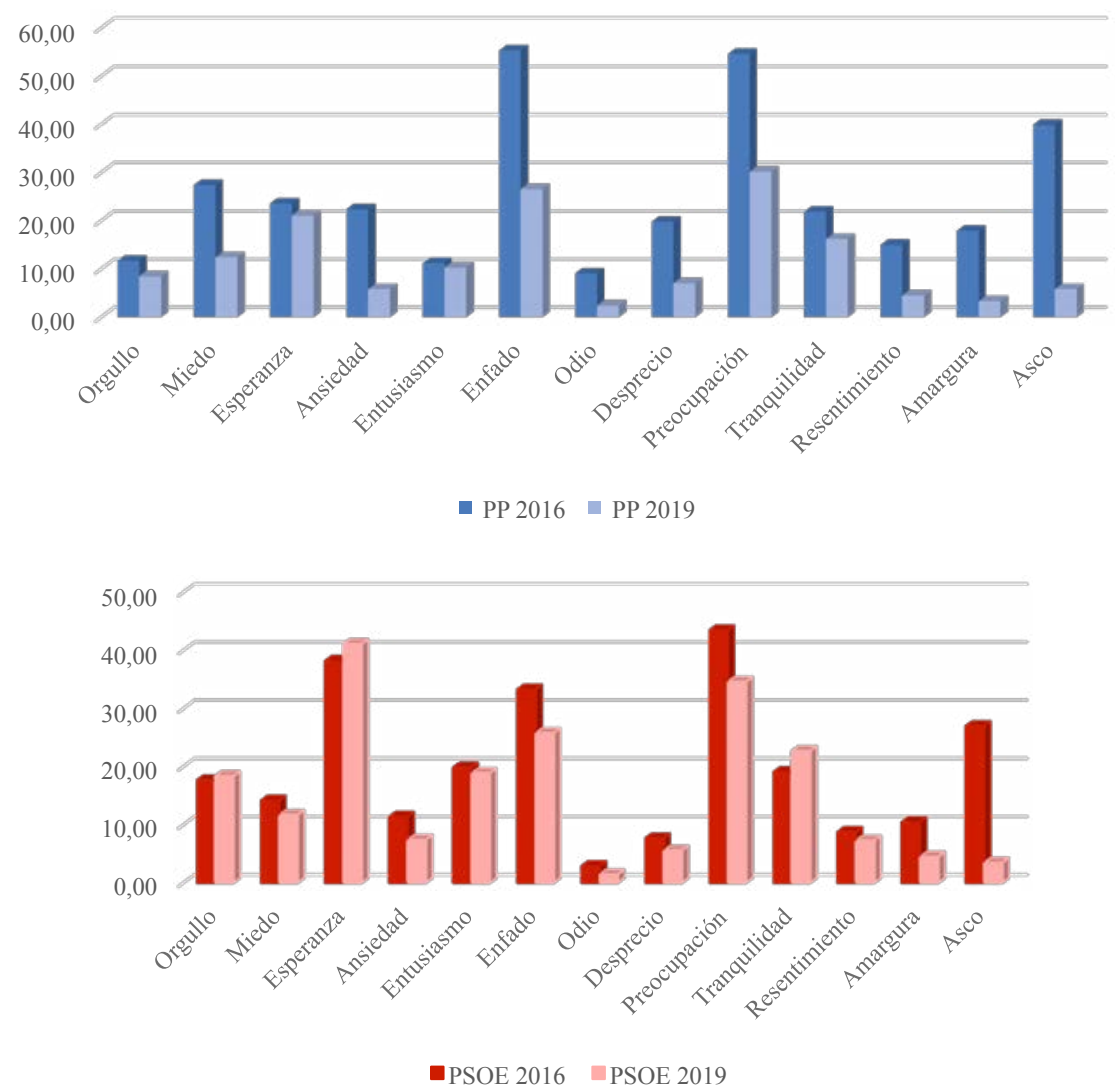


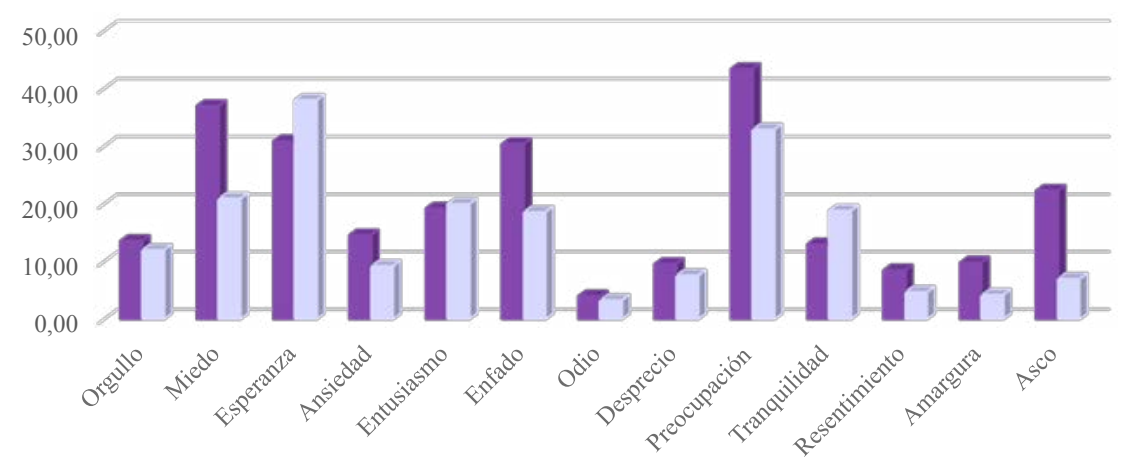

• Podemos 2016 Podemos 2019
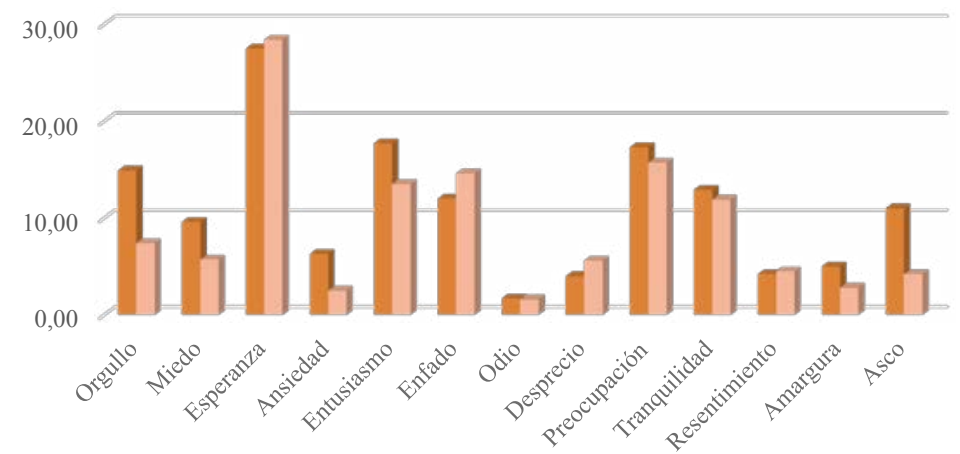

- Cs $2016 \square$ Cs 2019

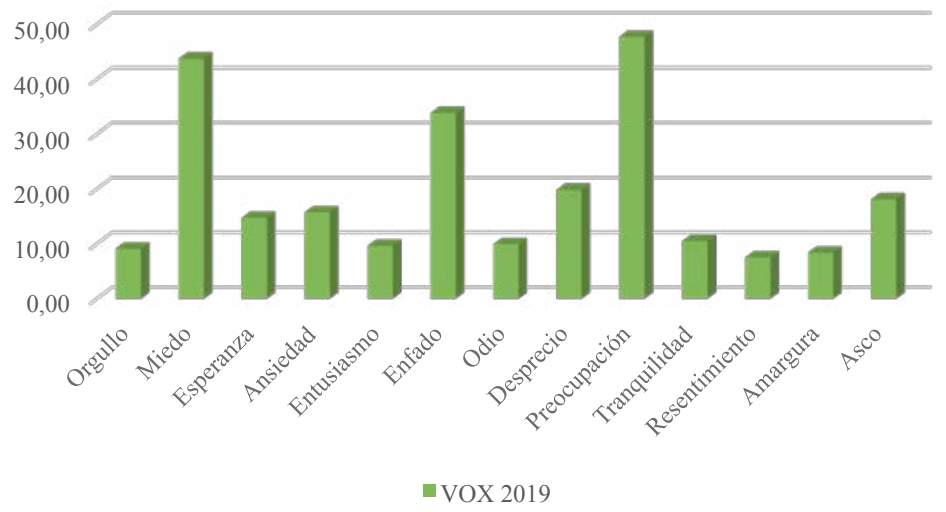

Fuente: elaboración propia a partir de datos de los estudios EPEGE. Emociones y comportamiento electoral 2016 y EPEGE nov.19 del EIP-USC. 
Es importante señalar que, al igual que sucede con el PSOE, sube en el año 2019 el porcentaje de aquellos que muestran sentir tranquilidad $(13 \%$ en $2016,18,8 \%$ en 2019) hacia esta formación, lo que sin duda podría tener relación con la diferente posición institucional de ambas formaciones en la actualidad.

Por su parte, Ciudadanos (Cs) es el único partido que junto con el PSOE muestra en ambos años como emoción con mayor presencia la esperanza (27,5\% en $2016,28,4 \%$ en 2019$)$, si bien es importante señalar que con porcentajes notablemente inferiores a los de la formación socialista y la formación morada. Sin duda, es también el partido que muestra mayores cambios entre ambos momentos temporales en lo que al conjunto de emociones se refiere, quizás fruto no solo de su propio devenir en términos de organización interna, sino también de los resultados obtenidos en ambas elecciones. Así, mientras podemos observar como del año 2016 al año 2019 cae notablemente el porcentaje de orgullo (14,9\% en $2016,7,4 \%$ en 2019$)$ y entusiasmo $(17,7 \%$ en $2016,13,5 \%$ en 2019$)$ hacia esta formación, sube el de una emoción tan negativa como el desprecio ( $4 \%$ en $2016,5,6 \%$ en 2019 ).

Finalmente, el caso de VOX es sin duda paradigmático: el miedo (43,7\%) y la preocupación $(47,7 \%)$ son las dos emociones que presentan mayores porcentajes, doblando en el caso de la primera el porcentaje del segundo partido que mayor presencia de esta emoción muestra y obteniendo, en el caso de la segunda, al menos 13 puntos porcentuales más que en el resto de formaciones. Estos resultados estarían en la línea de lo expuesto en los trabajos de Vasilopoulos et al. (2019) aplicados a los partidos de extrema derecha en Francia, en lo que al miedo se refiere, no así en el caso del enfado (33,8\%). En cuanto a esta emoción, esta formación presenta porcentajes dispares, si bien no tanto a los del resto de formaciones tradicionales (26,6\% PP, 25,8\% PSOE) y nuevas formaciones (18,6\% Podemos, $14,6 \% \mathrm{Cs})$; muy probablemente, las motivaciones que se encontrarían detrás de cada uno de ellos serían muy diferentes. Es también importante señalar, al contrario de lo observado en el resto de los partidos, los elevados porcentajes de presencia de emociones muy negativas como desprecio $(19,8 \%)$ y asco $(18,1 \%)$, siendo en ambos superior, al menos en 11 o 12 puntos porcentuales, a los obtenidos en estas emociones por el partido que se situaría en segundo lugar (Podemos).

$\mathrm{Si}$, en su defecto, observamos los datos de la presencia emocional hacia los líderes (gráfico 2), nos encontramos en un primer momento con una cuestión relevante, y es que, a simple vista y en líneas generales, se observan mayores porcentajes hacia los líderes que los observados hacia los partidos. Pero veamos algunas de estas cuestiones con mayor detalle.

En el caso del liderazgo del PP, y si bien es cierto que debemos tomar los datos con ciertos matices debido al cambio que se produjo entre ambos 
procesos al pasar de Mariano Rajoy a Pablo Casado, las emociones que en mayor medida despierta el liderazgo popular son las de enfado $(56,4 \%$ en $2016,30,5 \%$ en 2019$)$ y preocupación $(56,8 \%$ en $2016,34,2 \%$ en 2019$)$, reduciéndose considerablemente ambos porcentajes de 2016 a 2019. La emoción que de nuevo muestra un menor nivel de presencia es el odio $(8,9 \%$ en $2016,3,6 \%$ en 2019), reduciéndose el porcentaje entre ambos ańos. Es importante señalar que, si bien después del odio, en 2016 las dos emociones que presentaban menor porcentaje de presencia eran el entusiasmo y el orgullo, en 2019 son el resentimiento y la amargura. A pesar de todo, Pablo Casado es el líder que menor presencia emocional de orgullo y entusiasmo provoca, en algunos casos incluso con más de seis puntos y medio de diferencia respecto a Pablo Iglesias o Pedro Sánchez.

Gráficos 2. Presencia emocional líderes 2016-2019
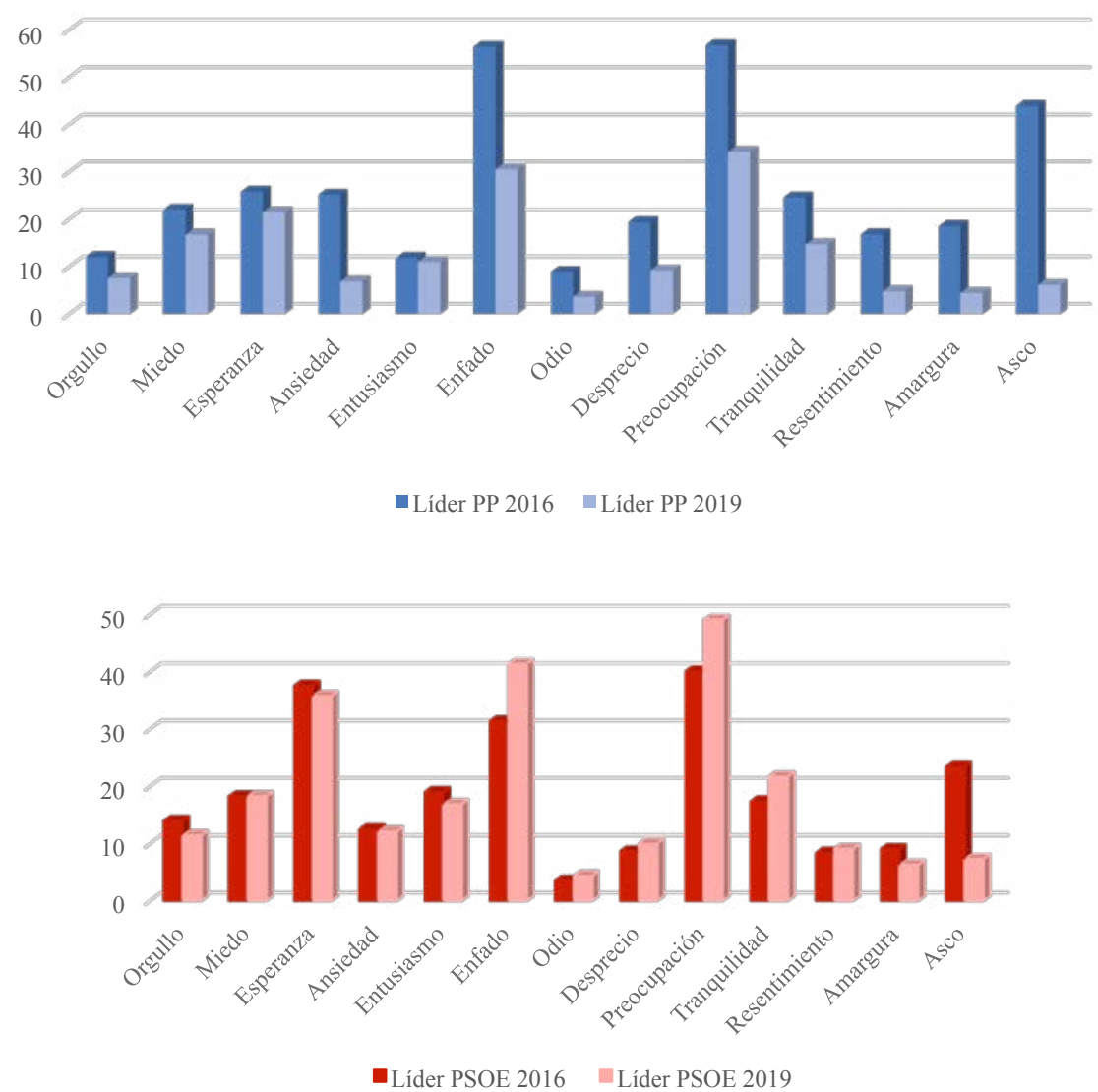


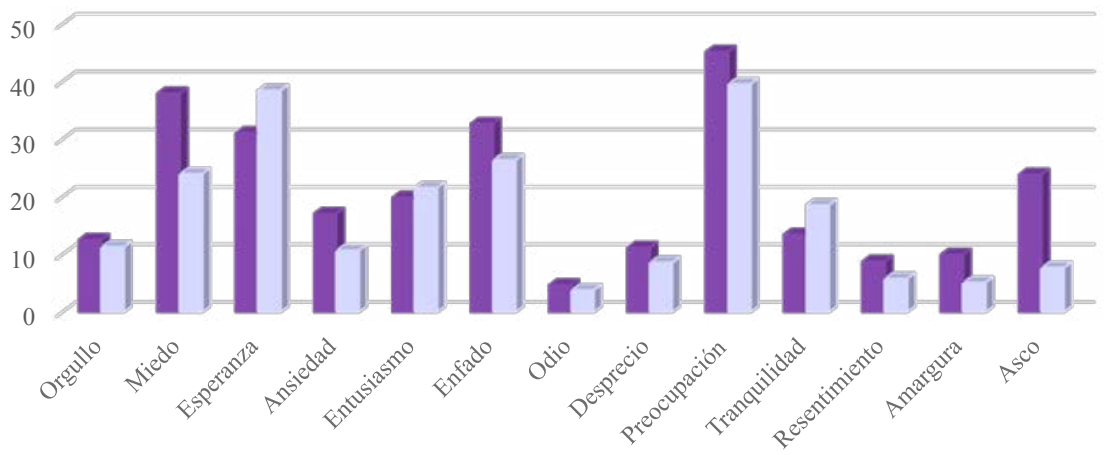

- Líder Podemos 2016 Líder Podemos 2019
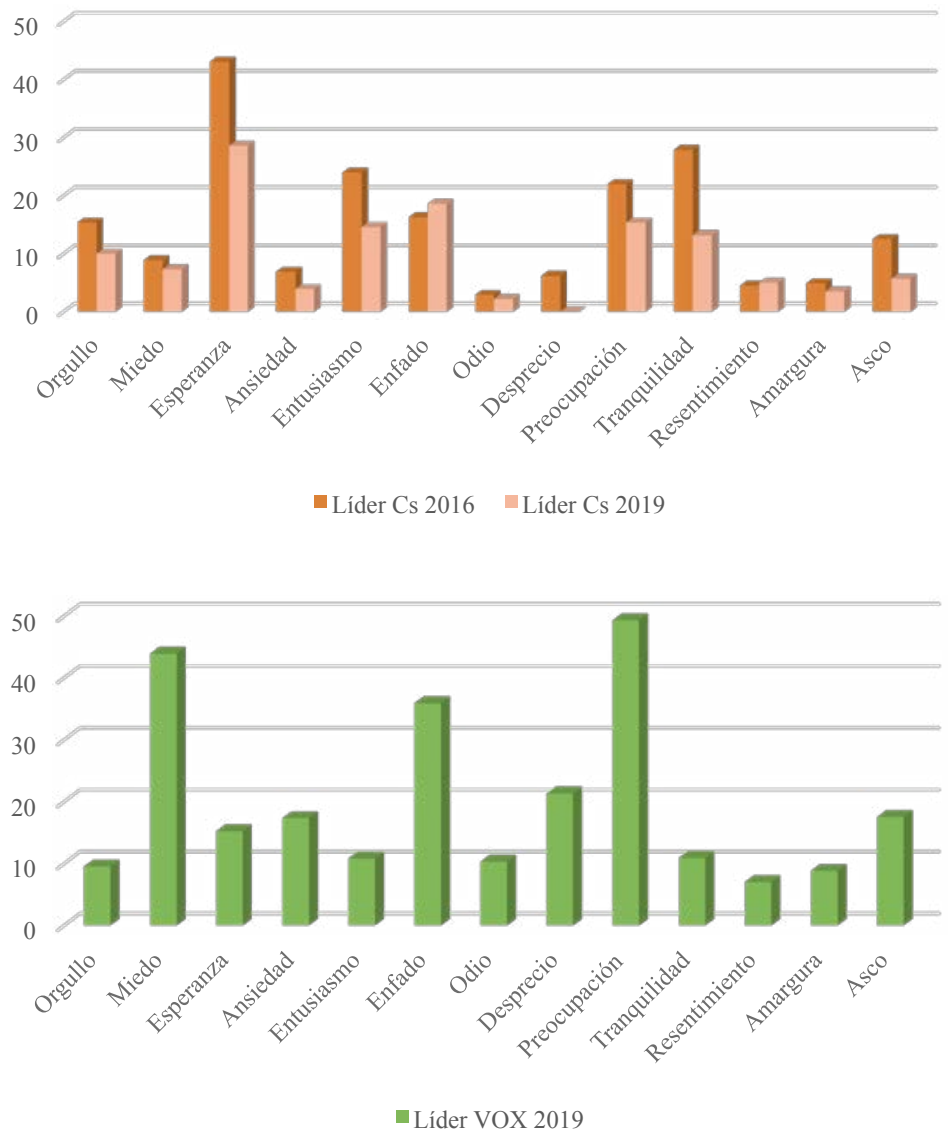

Fuente: elaboración propia a partir de datos de los estudios EPEGE. Emociones y comportamiento electoral 2016 y EPEGE nov.19 del EIP-USC. 
Si atendemos al liderazgo de Pedro Sánchez, observamos con claridad que las dos emociones con mayor presencia son, al igual que en el caso de su partido, la esperanza $(37,6 \%)$ y la preocupación $(40,1 \%)$ en el año 2016, produciéndose un cambio en el ańo 2019: la esperanza pasa a ser la tercera emoción en porcentaje $(35,8 \%)$, precedida por la preocupación $(49,1 \%)$ y el enfado $(41,4 \%)$. Al igual que en el caso del líder popular y en el caso de ambas formaciones, PSOE y PP, la emoción que en menor medida despierta el líder socialista es el odio (3,6\% en 2016, 4,4\% en 2019), seguida de cerca por emociones muy negativas como el resentimiento, el desprecio o la amargura. Como sucedía en el caso de la presencia de la formación socialista, volvemos a ver cómo entre ambos años se produce un crecimiento en el porcentaje de tranquilidad (17,4\% en $2016,21,7 \%$ en 2019$)$.

En el caso del líder de Podemos, Pablo Iglesias, observamos un cambio importante entre ambos momentos, similar a lo observado para su formación. Si en el año 2016 las dos emociones que mayor presencia presentaban eran la preocupación $(45,2 \%)$ y el miedo (38\%), en el año 2019 , si bien la preocupación permanece $(39,6 \%)$, aun perdiendo valor, el miedo es sustituido por una emoción positiva, la esperanza $(38,5 \%)$. Se mantiene el patrón observado en anteriores liderazgos respecto de la emoción que presenta menor presencia, el odio (4,7\% en $2016,3,8 \%$ en 2019). De nuevo, y al igual que sucedía en el caso de ambas formaciones de izquierda y en el líder socialista, volvemos a observar un importante crecimiento de la presencia de tranquilidad entre ambos momentos (13,5\% en $2016,18,6 \%$ en 2019).

Revelador tanto de los resultados obtenidos por su formación como de los cambios que se avecinaban, es la caída generalizada en la presencia emocional de Albert Rivera. Si bien en ambos años la emoción que mayor presencia muestra con importantes diferencias respecto al resto (algo que no ocurre de forma tan marcada en el resto de los líderes) es la esperanza, es notable el descenso de casi quince puntos porcentuales. También importante es la emoción que ocupa el segundo lugar, pues si en 2016 era la tranquilidad $(27,9 \%)$, en el 2019 pasa a ser el enfado $(18,6 \%)$. Al igual que en el resto de los casos, el odio sigue siendo la emoción que menor presencia muestra.

Para finalizar el análisis de la presencia emocional en los líderes, se muestra el caso del líder de VOX, Santiago Abascal, donde se replica el esquema observado para su formación, siendo la preocupación (49,3\%), el miedo $(43,9 \%)$ y el enfado $(35,9 \%)$ las tres emociones que mayor presencia muestran, con importantes diferencias respecto a los porcentajes obtenidos por otros líderes, especialmente en el caso del miedo. Por otro lado, y contrariamente a lo observado en el resto de los casos, el odio no es la emoción que menor presencia muestra (10,3\%), a lo cual se une el hecho de que sea el líder que menor porcentaje de presencia emocional positiva provoca, en algunos 
casos con importantes diferencias, salvo en el caso del entusiasmo (10,8\%), donde muestra un porcentaje prácticamente idéntico al de Casado (11\%).

\section{INTENSIDAD EMOCIONAL}

Analizada la presencia emocional, en el gráfico 3 pasamos a mostrar las intensidades medias con las que los entrevistados han dicho haber sentido las emociones. De nuevo, haremos un análisis diferenciado para líderes y partidos.

En el caso del PP no existe un patrón respecto a las emociones que en ambos ańos presentan mayores y menores niveles de intensidad. Así, si en 2016 las mayores intensidades son las que muestran el enfado $(4,35)$ y el odio $(4,23)$, en 2019 son sustituidas por asco $(4,08)$, resentimiento $(3,99)$ y amargura $(3,98)$. En el caso de las emociones con intensidades medias más bajas, en 2016 estas son tranquilidad $(3,58)$, entusiasmo $(3,71)$ y esperanza $(3,74)$; mientras que en 2019 son ansiedad $(3,38)$, entusiasmo $(3,42)$ y miedo $(3,47)$. Especialmente preocupante son los niveles de intensidad de las emociones positivas en 2016, pues como hemos comprobado en otros trabajos (Parga y Pereira, 2019) las intensidades de las emociones positivas tienden a ser soportadas por los votantes identificados, y en el caso del PP esta tendencia no se estaría produciendo en demasía.

Por su parte, al igual que en el caso anterior, en el PSOE tampoco se observa un patrón claro. Si en 2016 las emociones con mayores niveles de intensidad eran enfado $(3,87)$ y miedo $(3,75)$, en 2019 serán odio $(4,67)$, amargura $(4,26)$ y desprecio $(4,21)$. Las emociones con menores niveles de intensidad eran en 2016 tranquilidad $(3,31)$ y resentimiento $(3,48)$ y en 2019 , si bien se mantendrá la tranquilidad $(3,53)$, aparecerá el entusiasmo $(3,39)$.

Frente a los dos casos anteriores, sí observamos algunos patrones en el caso de Podemos, pues en ambos años la emoción con mayor nivel de intensidad es el odio (4,30 en 2016, 4,61 en 2019), seguida en 2016 del miedo $(4,10)$ y en 2019 de la amargura $(4,53)$. También se observa un patrón común para el caso de la emoción con menor intensidad, la tranquilidad $(3,22$ en $2016,3,39$ en 2019), seguida de la amargura en $2016(3,38)$ y del entusiasmo en 2019 (3,45).

En el caso de Ciudadanos, al igual que en el caso de la formación morada, también observamos un patrón, que además ambas comparten respecto a la emoción que mayor intensidad media presenta en ambos años, el odio (4,76 en 2016, 4,61 en 2019), seguida del desprecio $(4,20)$ en 2016 y el asco $(4,30)$ en 2019. No observamos este patrón respecto a las emociones que menor intensidad reflejan, siendo la esperanza en $2016(3,52)$ y el entusiasmo en $2019(3,24)$.

Por último, en la formación de extrema derecha, VOX, observamos cómo las intensidades medias de todas las emociones son más altas que las del resto, situándose en todos los casos por encima del 4 (sobre 5), salvo en el caso 
de dos emociones positivas, entusiasmo $(3,68)$ y tranquilidad $(3,81)$. Las emociones que presentan intensidades medias más elevadas son tres de las que conforman el componente emocional muy negativo: desprecio $(4,51)$, resentimiento $(4,50)$ y asco $(4,50)$.

\section{Gráficos 3. Intensidad emocional partidos 2016-2019}
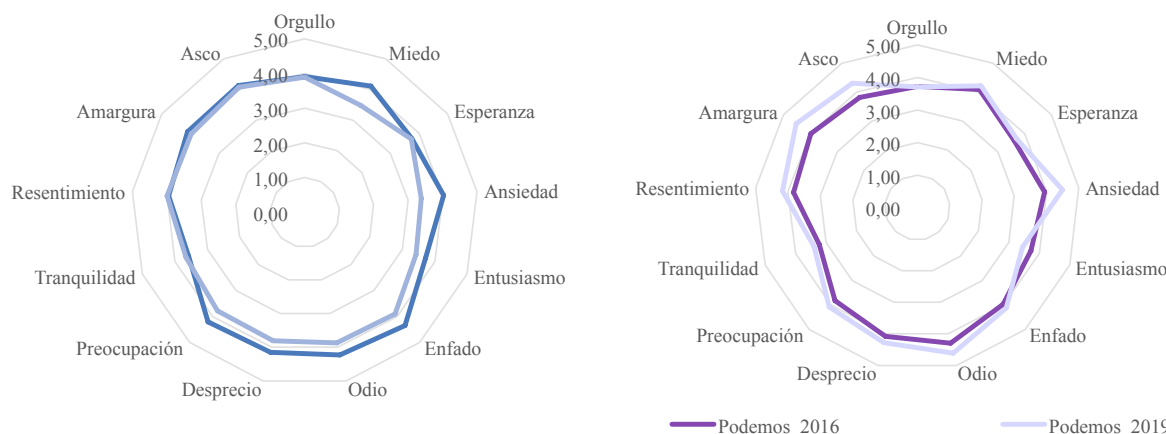

-PP $2016 \quad$ PP 2019
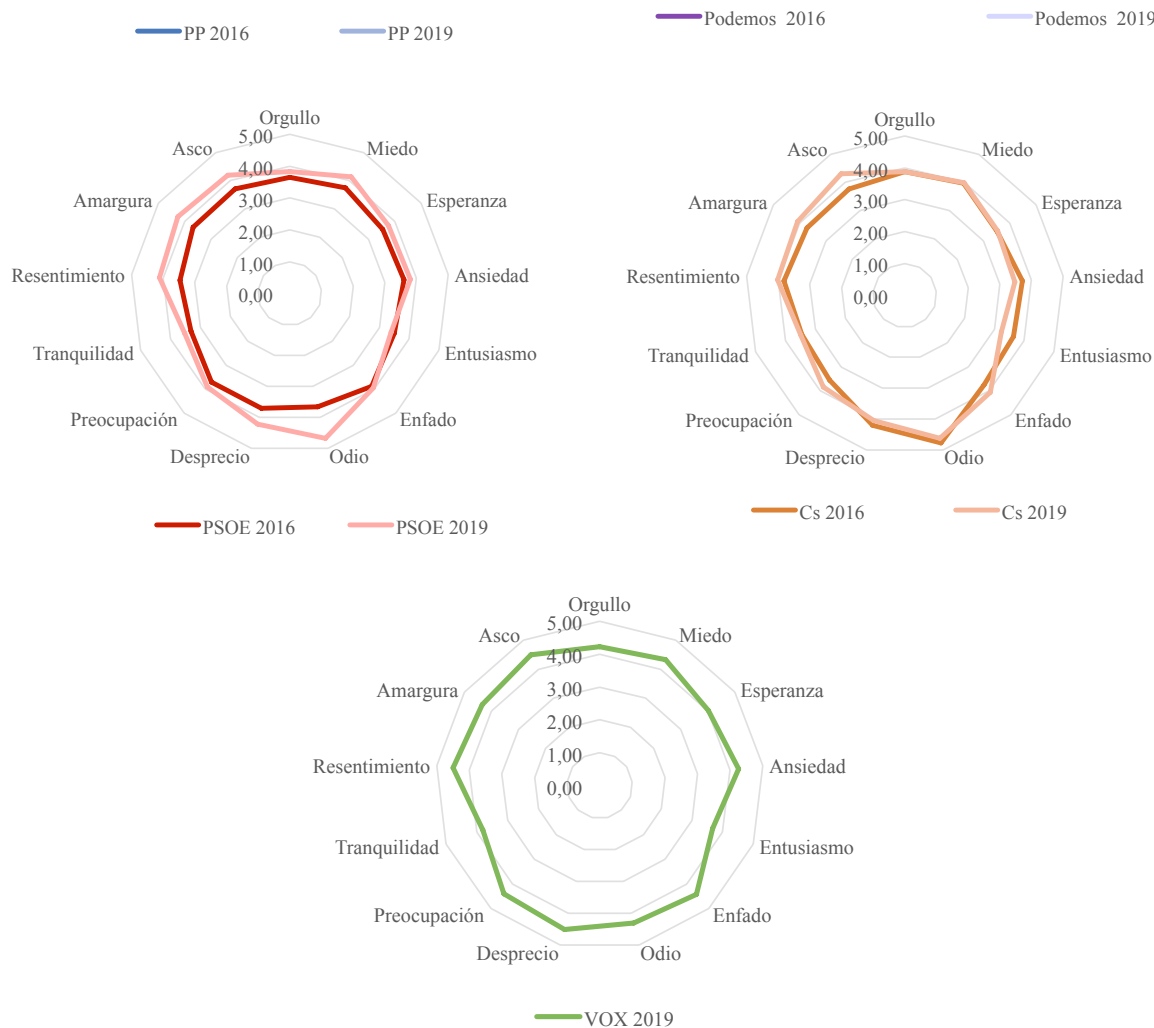

Fuente: elaboración propia a partir de datos de los estudios EPEGE. Emociones y comportamiento electoral 2016 y EPEGE nov.19 del EIP-USC. 
A continuación, observaremos las intensidades emocionales hacia los líderes de los cinco partidos (gráfico 4). En líneas generales, y al igual que en el caso de los partidos políticos, las intensidades de los cuatro líderes para los que hay datos en ambos estudios son superiores en todas las emociones en 2019, con contadas excepciones, si bien en el caso del líder del PP todas las intensidades son menores (salvo en dos), lo que pudiera explicarse por el cambio de liderazgo. Esto nos permitiría afirmar que las elecciones de noviembre de 2019 generaron un clima de mayor tensión emocional.

Como señalábamos anteriormente, las mayores diferencias entre líderes y tiempo las encontramos en el caso del liderazgo del PP, más que posiblemente provocado por el cambio de líder que se produjo entre ambos procesos. Aun así, cabe señalar que en los dos años la emoción que mayor intensidad muestra es el enfado (4,18 en 2016, 4,04 en 2019), seguida de la preocupación en $2016(4,06)$ y del resentimiento en $2019(3,87)$. Por su parte, las intensidades más bajas no presentan un patrón: si en 2016 eran la ansiedad $(3,65)$, la esperanza y la tranquilidad (ambas con 3,69), en 2019 era el entusiasmo $(3,25)$.

En el caso del líder del partido socialista, Pedro Sánchez, las intensidades no muestran un patrón entre ambos momentos, quizás fruto de su cambio de posición institucional, aunque sí replica el patrón en 2016 respecto a las intensidades emocionales expresadas hacia el partido; no así en 2019, donde parece haber un desligamiento más claro entre formación y liderazgo. Así, si en 2016 las emociones con mayor intensidad eran enfado $(3,92)$ y miedo $(3,80)$, en 2019 son sustituidas por desprecio y amargura (ambas con 4,12), mientras que las más bajas en 2016 eran resentimiento $(3,33)$ y tranquilidad $(3,43)$ y en 2019 lo eran tranquilidad $(3,50)$ y entusiasmo $(3,60)$.

Por su parte, Pablo Iglesias, al igual que en el caso del líder socialista, muestra unas intensidades emocionales similares a las expresadas por su formación en ambos años, con alguna excepción. Así, el miedo $(4,11)$ es junto a la amargura $(4,08)$ las emociones que mayor intensidad mostraban en 2016, mientras que en 2019 lo eran el odio $(4,42)$ y el desprecio $(4,41)$. Las emociones de menor intensidad eran en 2016 la tranquilidad $(3,09)$, al igual que en 2019 (3,43), uniéndose el entusiasmo $(3,41)$.

Albert Rivera, si bien no replica el patrón de intensidad emocional observado en su partido, sí muestra una continuidad entre ambos momentos analizados. Así, tanto en 2016 como en 2019 una de las emociones que mayor intensidad refleja es el odio (3,79 en 2016, 4,29 en 2019), a la que acompañan en 2016 el enfado $(3,81)$ y en 2019 el asco $(4,17)$. Las emociones con menores niveles de intensidad son en 2016 la tranquilidad $(3,26)$ y el resentimiento $(3,27)$, y en 2019 el entusiasmo $(3,62)$ y la ansiedad $(3,65)$. Es importante señalar una cuestión: el patrón descrito hace que sea uno de los líderes 
que, junto a su formación, estaría completando el abanico de intensidades emocionales negativas, lo que, sin duda, iría en claro perjuicio de su éxito electoral y explicaría los resultados obtenidos por esta formación en 2019, así como el deterioro de su liderazgo.

\section{Gráficos 4. Intensidad emocional lideres 2016-2019}
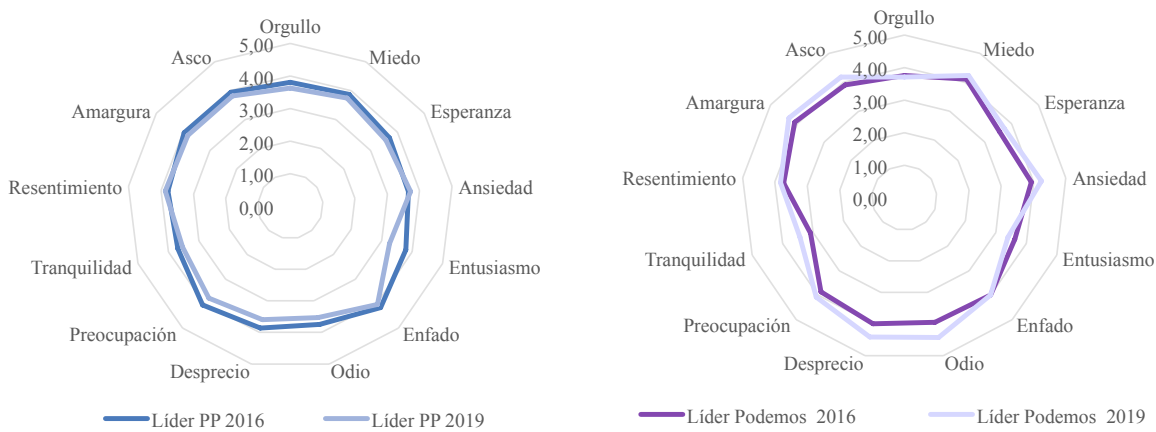

—Líder PP 2016 L Líder PP 2019
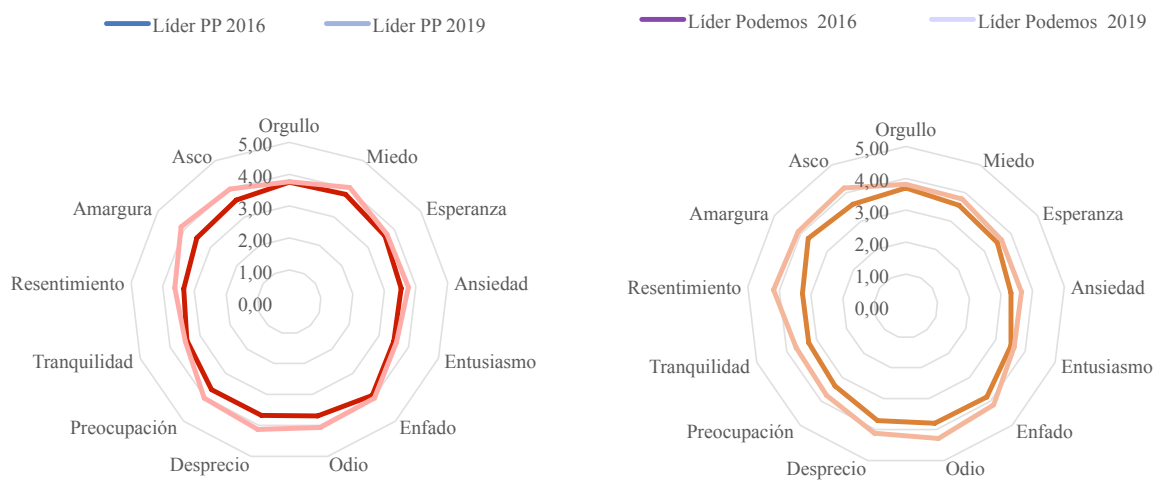

—Líder PSOE 2016 Líder PSOE 2019
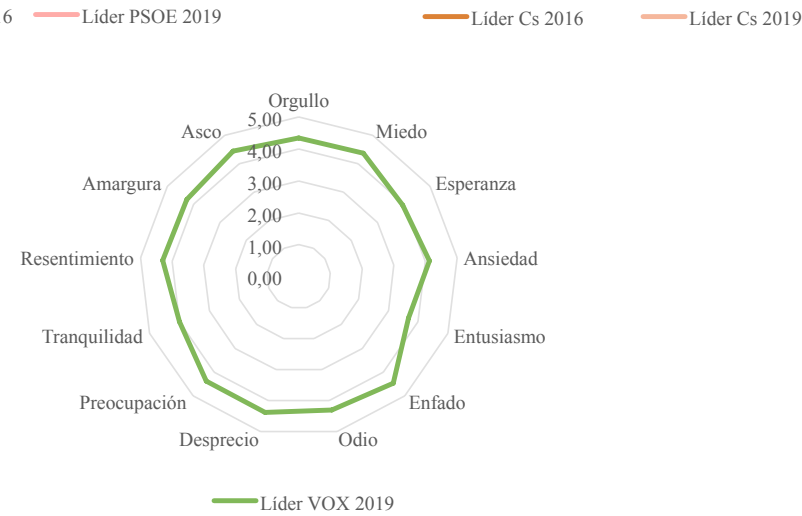

Fuente: elaboración propia a partir de datos de los estudios EPEGE. Emociones y comportamiento electoral 2016 y EPEGE nov.19 del EIP-USC. 
En el caso de Santiago Abascal, sus intensidades son mayores que las observadas en otros líderes, lo que hace pensar en una mayor uniformidad de criterio entre ciudadanos de distintos perfiles. Las emociones que mayores niveles de intensidad reflejan son el enfado $(4,46)$ y el asco $(4,44)$, y las más bajas el entusiasmo $(3,67)$ y la esperanza $(3,95)$.

\section{PERSISTENCIA EMOCIONAL}

Para cerrar este análisis descriptivo, mostramos los niveles de persistencia emocional para cada uno de los partidos políticos y sus líderes (tabla 2). En líneas generales, y teniendo en cuenta la batería de emociones planteada, los niveles de persistencia son notablemente elevados en ambos supuestos, independientemente de si atendemos a formaciones o líderes, aunque ligeramente superiores en el año 2016 que en el 2019. Esto último podría venir explicado no solo por la presencia de nuevos actores en la escena política, sino también

Tabla 2. Persistencia emocional partidos políticos 2016-2019

\begin{tabular}{lrrrrrrrrr}
\hline & \multicolumn{3}{c}{ PP } & \multicolumn{2}{c}{ PSOE } & \multicolumn{1}{c}{ PODEMOS } & Cs & VOX \\
\cline { 2 - 11 } & $\mathbf{2 0 1 6}$ & $\mathbf{2 0 1 9}$ & $\mathbf{2 0 1 6}$ & $\mathbf{2 0 1 9}$ & $\mathbf{2 0 1 6}$ & $\mathbf{2 0 1 9}$ & $\mathbf{2 0 1 6}$ & $\mathbf{2 0 1 9}$ & $\mathbf{2 0 1 9}$ \\
\hline Orgullo & 85,7 & 73,7 & 100,0 & 65,5 & 88,9 & 84,3 & 100,0 & 81,6 & 100,0 \\
\hline Miedo & 93,3 & 91,5 & 100,0 & 91,1 & 100,0 & 94,0 & 100,0 & 82,3 & 97,6 \\
\hline Esperanza & 90,9 & 78,7 & 87,5 & 74,5 & 84,6 & 81,9 & 85,7 & 52,6 & 98,0 \\
\hline Ansiedad & 100,0 & 94,8 & 100,0 & 86,4 & 100,0 & 97,6 & 100,0 & 80,5 & 99,4 \\
\hline Entusiasmo & 100,0 & 77,8 & 85,7 & 75,1 & 84,6 & 82,8 & 100,0 & 59,8 & 96,9 \\
\hline Enfado & 100,0 & 92,9 & 100,0 & 83,9 & 100,0 & 91,0 & 100,0 & 87,3 & 98,5 \\
\hline Odio & 100,0 & 96,0 & - & 93,8 & 100,0 & 97,0 & - & 93,8 & 100,0 \\
\hline Desprecio & 92,9 & 91,5 & 100,0 & 87,3 & 100,0 & 98,8 & 100,0 & 89,4 & 99,5 \\
\hline Preocupación & 100,0 & 95,3 & 100,0 & 93,9 & 100,0 & 95,7 & 100,0 & 87,7 & 98,0 \\
\hline Tranquilidad & 100,0 & 77,9 & 100,0 & 81,9 & 100,0 & 92,9 & 83,3 & 76,7 & 100,0 \\
\hline Resentimiento & 100,0 & 92,9 & 100,0 & 91,8 & 50,0 & 95,8 & - & 93,5 & 100,0 \\
\hline Amargura & 100,0 & 93,8 & 100,0 & 97,8 & 100,0 & 100,0 & 100,0 & 96,6 & 98,8 \\
\hline Disgusto/Asco & 100,0 & 100,0 & 100,0 & 93,7 & 100,0 & 100,0 & 100,0 & 90,9 & 98,2 \\
\hline
\end{tabular}

Fuente: elaboración propia a partir de datos de los estudios EPEGE. Emociones y comportamiento electoral 2016 y EPEGE nov.19 del EIP-USC. 
por el cambio de posición institucional de algunos y, en consecuencia, la aparición de nuevas emociones todavía no asentadas.

En el caso de los partidos políticos podemos observar cómo, en líneas generales, son las emociones relacionadas con la ansiedad o la aversión aquellas que muestran mayores niveles de persistencia, independientemente del partido al que hagamos referencia; sin embargo, es curioso que las emociones positivas tengan elevados niveles de prevalencia en el caso de VOX, como ocurre con el orgullo. En el caso de la persistencia hacia los líderes, los porcentajes siguen un patrón similar al observado en el caso de los partidos: de nuevo son las emociones con carga negativa o muy negativa las que muestran mayores valores en prácticamente todos los casos. Volvemos a encontrar la misma excepción en el caso del líder de VOX, el único que muestra un nivel de persistencia del $100 \%$ en alguna emoción positiva (orgullo). En cualquier caso, debemos ser conscientes de que los porcentajes de prevalencia son en

Tabla 3. Persistencia emocional líderes politicos 2016-2019

\begin{tabular}{lrrrrrrrrrr}
\hline & \multicolumn{2}{c}{ Líder PP } & \multicolumn{2}{c}{ Líder PSOE } & \multicolumn{2}{c}{$\begin{array}{c}\text { Líder } \\
\text { PODEMOS }\end{array}$} & Líder Cs & $\begin{array}{c}\text { Líder } \\
\text { VOX }\end{array}$ \\
\cline { 2 - 12 } & $\mathbf{2 0 1 6}$ & $\mathbf{2 0 1 9}$ & $\mathbf{2 0 1 6}$ & $\mathbf{2 0 1 9}$ & $\mathbf{2 0 1 6}$ & $\mathbf{2 0 1 9}$ & $\mathbf{2 0 1 6}$ & $\mathbf{2 0 1 9}$ & $\mathbf{2 0 1 9}$ \\
\hline Orgullo & 66,7 & 94,6 & 100,0 & 82,0 & 80,0 & 93,8 & 100,0 & 66,5 & 100,0 \\
\hline Miedo & 100,0 & 88,3 & 90,9 & 91,7 & 93,3 & 94,9 & 75,0 & 56,5 & 96,3 \\
\hline Esperanza & 92,9 & 88,7 & 88,9 & 89,4 & 85,7 & 85,0 & 95,2 & 29,7 & 97,3 \\
\hline Ansiedad & 90,0 & 89,3 & 80,0 & 88,9 & 100,0 & 97,0 & 100,0 & 61,4 & 98,2 \\
\hline Entusiasmo & 100,0 & 92,2 & 72,7 & 92,2 & 76,9 & 79,8 & 100,0 & 37,4 & 99,1 \\
\hline Enfado & 88,9 & 90,9 & 100,0 & 79,7 & 100,0 & 86,8 & 100,0 & 68,5 & 97,0 \\
\hline Odio & 100,0 & 84,5 & - & 92,6 & 100,0 & 100,0 & 100,0 & 81,5 & 98,0 \\
\hline Desprecio & 100,0 & 93,3 & 100,0 & 97,8 & 100,0 & 100,0 & 100,0 & 74,7 & 98,6 \\
\hline Preocupación & 100,0 & 92,4 & 91,3 & 91,7 & 96,0 & 93,9 & 100,0 & 59,9 & 97,9 \\
\hline Tranquilidad & 100,0 & 89,7 & 80,0 & 94,4 & 90,0 & 95,7 & 100,0 & 59,4 & 97,7 \\
\hline Resentimiento & 100,0 & 95,2 & 100,0 & 93,2 & 100,0 & 94,4 & 100,0 & 93,7 & 100,0 \\
\hline Amargura & 100,0 & 95,6 & 100,0 & 97,0 & 100,0 & 96,1 & 100,0 & 70,7 & 100,0 \\
\hline Disgusto/Asco & 89,3 & 100,0 & 100,0 & 98,7 & 100,0 & 98,4 & 100,0 & 85,1 & 98,3 \\
\hline
\end{tabular}

Fuente: elaboración propia a partir de datos de los estudios EPEGE. Emociones y comportamiento electoral 2016 y EPEGE nov.19 del EIP-USC. 
prácticamente todos los casos muy elevados, si bien es importante comentar algunas excepciones. En este sentido, una de las más importantes es la que representan tanto los niveles de persistencia de Cs como los de su líder Albert Rivera, especialmente en el año 2019, donde observamos una mayor variabilidad en los datos (tabla 3). En ambos casos y en términos comparados al resto de formaciones y líderes, se presentan bajos niveles de persistencia en lo que a las emociones positivas se refiere: sirvan de ejemplo el 52,6\% de esperanza o el 59,8\% de entusiasmo hacia el partido; o bien el 29,7\% de esperanza, el $37,4 \%$ de entusiasmo y el 59,4\% de tranquilidad hacia el líder. Sin duda, como veníamos exponiendo, estos datos serían reveladores de los resultados de la formación y de sus propios problemas internos y de liderazgo.

\section{CONSTRUCCIÓN DE COMPONENTES EMOCIONALES}

Para cerrar el análisis descriptivo, y puesto que como mencionamos en el apartado de metodología nuestro diseño parte no solo de una concepción individual, sino también agregada de la construcción emocional, y dado que, además, en las siguientes páginas realizaremos un análisis multivariante de esta segunda visión, presentamos a continuación un análisis descriptivo según componentes emocionales.

En la tabla 4 podemos observar los porcentajes medios de presencia emocional ${ }^{15}$ para las formaciones y líderes de forma agregada ${ }^{16}$. El componente emocional aversión es el que presenta en ambos años y sin distinción de objeto - partido o líderes- menores porcentajes; por el contrario, el componente ansiedad es el que presenta mayores porcentajes medios en 2016 en todos los partidos a excepción de Cs, que presentaba mayores niveles del componente entusiasmo. Sin embargo, podemos observar cómo este patrón cambia en 2019; así, tanto PSOE como Podemos obtienen mayores porcentajes de presencia emocional en el componente entusiasmo, no así en el caso del PP o de VOX. Este último muestra, además, un alto porcentaje en el componente ansiedad.

15 Estos valores han sido calculados a partir de los porcentajes medios de presencia emocional expresados para cada una de las emociones que conforman cada componente (ver apartado 3).

16 Nos gustaría hacer constar que, dado que entre ambos estudios se produce un cambio sustancial respecto a una de las emociones estudiada, que como ya se mencionó procede de las múltiples posibilidades de traducción que el vocablo en inglés permite (disgust = disgusto o asco), para el cálculo de esta agregación no se ha tenido en cuenta en el año 2016 el porcentaje de la variable disgusto. En el año 2019 sí se ha tenido en cuenta el porcentaje de la variable asco, pero dentro del cálculo para el componente emocional aversión. 
Tabla 4. Porcentajes medios de presencia emocional hacia partidos y lideres según componentes emocionales

\begin{tabular}{|c|c|c|c|c|c|}
\hline & \multicolumn{2}{|c|}{ Partidos } & \multicolumn{2}{|c|}{ Líderes } \\
\hline & & 2016 & 2019 & 2016 & 2019 \\
\hline \multirow{5}{*}{ Entusiasmo } & PP & 17,10 & 14,03 & 18,48 & 13,68 \\
\hline & PSOE & 23,73 & 25,28 & 22 & 21,45 \\
\hline & PODEMOS & 19,18 & 22,20 & 19,28 & 22,50 \\
\hline & Cs & 18,25 & 15,30 & 27,60 & 16,60 \\
\hline & VOX & & 10,98 & & 11,60 \\
\hline \multirow{5}{*}{ Ansiedad } & PP & 39,93 & 18,75 & 40,20 & 22,05 \\
\hline & PSOE & 25,60 & 19,88 & 25,58 & 30,23 \\
\hline & PODEMOS & 31,35 & 20,40 & 33,25 & 25,18 \\
\hline & Cs & 11,30 & 9,63 & 13,53 & 11,30 \\
\hline & VOX & & 35,23 & & 36,60 \\
\hline \multirow{5}{*}{ Aversión } & PP & 15,45 & 4,60 & 15,90 & 5,62 \\
\hline & PSOE & 7,48 & 4,48 & 7,45 & 7,42 \\
\hline & PODEMOS & 7,98 & 5,36 & 8,68 & 6,20 \\
\hline & Cs & 3,73 & 3,74 & 4,63 & 4,10 \\
\hline & VOX & & 12,74 & & 12,98 \\
\hline
\end{tabular}

Fuente: elaboración propia a partir de datos de los estudios EPEGE. Emociones y comportamiento electoral 2016 y EPEGE nov.19 del EIP-USC.

En el caso de los líderes, observamos cómo si bien se mantiene el patrón ya mencionado para el componente aversión, no sucede lo mismo con el resto de los componentes; así, en el caso del componente ansiedad observamos cómo Pedro Sánchez es el único líder que ve aumentar este porcentaje, frente al resto de líderes, que lo ve reducido. Con el componente emocional entusiasmo sucede a la inversa, es Sánchez el único líder que ve reducido su porcentaje en 2019.

Si atendemos a la comparación formación-líder, si bien en el caso del PP el componente ansiedad es mayor en ambos años en el caso del líder, no sucede igual en el caso de los otros dos componentes, siendo mayor en el líder el de entusiasmo en 2016 y el de aversión en 2019. En el caso de Pedro Sánchez y el PSOE, la presencia es en los tres componentes mayor para el líder en el año 2019, algo 
que también sucede en el caso de Pablo Iglesias en ambos años, salvo en el componente de entusiasmo, así como también en Santiago Abascal. Paradigmático es el caso del tándem Ciudadanos-Albert Rivera, donde en ambos años el porcentaje de presencia emocional es mayor en el líder en los tres componentes.

Por último, se presentan en la tabla 5 las intensidades medias de cada componente emocional hacia cada partido y líder. Observamos un patrón común a todos los tándems, independientemente del año, y es el hecho de que la intensidad del componente aversión recae siempre en mayor medida sobre la formación que sobre el líder. Por otro lado, en el caso de los nuevos partidos o en aquellos donde ha habido un cambio de liderazgo, la intensidad positiva (entusiasmo) se recoge en mayor medida hacia la formación que hacia el líder. Otra cuestión de gran interés son los casos donde una mayor presencia

\section{Tabla 5. Valores medios de intensidad emocional hacia partidos $y$ lideres según componentes emocionales}

\begin{tabular}{|c|c|c|c|c|c|}
\hline & & \multicolumn{2}{|c|}{ Partidos } & \multicolumn{2}{|c|}{ Líderes } \\
\hline & & 2016 & 2019 & 2016 & 2019 \\
\hline \multirow{5}{*}{ Entusiasmo } & $\mathrm{PP}$ & 3,74 & 3,67 & 3,74 & 3,50 \\
\hline & PSOE & 3,50 & 3,63 & 3,57 & 3,64 \\
\hline & PODEMOS & 3,57 & 3,56 & 3,50 & 3,57 \\
\hline & Cs & 3,63 & 3,54 & 3,49 & 3,68 \\
\hline & VOX & & 3,93 & & 3,99 \\
\hline \multirow{5}{*}{ Ansiedad } & $\mathrm{PP}$ & 4,17 & 3,64 & 3,94 & 3,82 \\
\hline & PSOE & 3,73 & 3,95 & 3,74 & 3,97 \\
\hline & PODEMOS & 3,96 & 4,23 & 3,97 & 4,14 \\
\hline & Cs & 3,75 & 3,84 & 3,51 & 3,83 \\
\hline & VOX & & 4,36 & & 4,33 \\
\hline \multirow{5}{*}{ Aversión } & $\mathrm{PP}$ & 4,10 & 3,91 & 3,84 & 3,73 \\
\hline & PSOE & 3,63 & 4,31 & 3,56 & 3,98 \\
\hline & PODEMOS & 4,05 & 4,40 & 3,93 & 4,23 \\
\hline & Cs & 4,12 & 4,19 & 3,62 & 4,17 \\
\hline & VOX & & 4,41 & & 4,34 \\
\hline
\end{tabular}

Fuente: elaboración propia a partir de datos de los estudios EPEGE. Emociones y comportamiento electoral 2016 y EPEGE nov.19 del EIP-USC. 
emocional redunda en mayores niveles de intensidad; es el caso del líder socialista, que en 2019 presenta mayores intensidades en los tres componentes que su partido. El caso contrario lo podemos observar en Albert Rivera para el año 2016, donde su partido presenta para los tres componentes mayores intensidades que él. Los casos de Podemos y PP presentan una mayor variación.

Para completar el análisis descriptivo presentado en páginas precedentes y con el objetivo de testar en nuestros casos de estudio los planteamientos de Marcus et al. $(2006,2017)$ se realizó un análisis factorial exploratorio ${ }^{17}$ de los tres componentes emocionales: entusiasmo, ansiedad y aversión. Así mismo, y para confirmar la validez interna de estos tres componentes latentes, se incluyó también un análisis de fiabilidad. Como se puede observar en los resultados, tablas 6 y 7, los factores extraídos agrupan el total de las emociones incluidas en el estudio, salvo alguna excepción que comentaremos, permitiendo diferenciar los tres componentes mencionados. Ambos análisis han sido realizados teniendo en cuenta los presupuestos estadísticos que ambas técnicas requieren, por lo que únicamente se ha trabajado con las variables de intensidad emocional.

Tabla 6. Análisis factorial y análisis de fiabilidad de los componentes emocionales hacia los partidos politicos 2016-201918

\begin{tabular}{|c|c|c|c|c|c|c|c|c|c|c|}
\hline & & \multicolumn{2}{|c|}{ PP } & \multicolumn{2}{|c|}{ PSOE } & \multicolumn{2}{|c|}{ PODEMOS } & \multicolumn{2}{|c|}{ Cs } & \multirow{2}{*}{$\begin{array}{l}\text { VOX } \\
2019\end{array}$} \\
\hline & & 2016 & 2019 & 2016 & 2019 & 2016 & 2019 & 2016 & 2019 & \\
\hline $\begin{array}{l}\text { Factor } \\
\text { Entusiasmo }\end{array}$ & \multirow{2}{*}{$\begin{array}{l}\text { Esperanza } \\
\text { Entusiasmo } \\
\text { Orgullo } \\
\text { Tranquilidad }\end{array}$} & 70,08 & 74,43 & 74,50 & 72,52 & 71,75 & 76,26 & 78,37 & 74,19 & 75,97 \\
\hline $\begin{array}{l}\text { Alfa de } \\
\text { Cronbach }\end{array}$ & & 0,854 & 0,883 & 0,878 & 0,871 & 0,859 & 0,887 & 0,907 & 0,880 & 0,868 \\
\hline $\begin{array}{l}\text { Factor } \\
\text { Ansiedad }\end{array}$ & \multirow{2}{*}{$\begin{array}{l}\text { Miedo } \\
\text { Preocupación } \\
\text { Enfado } \\
\text { Ansiedad }\end{array}$} & 72,90 & 77,92 & 70,42 & 64,39 & 72,88 & - & 74,83 & 86,88 & 56,85 \\
\hline $\begin{array}{l}\text { Alfa de } \\
\text { Cronbach }\end{array}$ & & 0,825 & 0,905 & 0,888 & 0,776 & 0,905 & - & 0,914 & 0,942 & 0,731 \\
\hline
\end{tabular}

17 Para el análisis factorial se ha utilizado la rotación ortogonal varimax y el método de extracción de factores por componentes principales.

18 Tal y como ya se mencionó en el análisis descriptivo y con el fin de facilitar la comparación y ateniéndonos a la versión definitiva de la batería de emociones manejada (2019), el análisis multivariante en el año 2016 obvió la emoción disgusto y en el análisis de 2019 sí se incluyó la emoción de asco en el componente aversión, tal y como se puede observar en las tablas 6 y 7. 


\begin{tabular}{|c|c|c|c|c|c|c|c|c|c|c|}
\hline & & \multicolumn{2}{|c|}{ PP } & \multicolumn{2}{|c|}{ PSOE } & \multicolumn{2}{|c|}{ PODEMOS } & \multicolumn{2}{|c|}{ Cs } & \multirow{2}{*}{$\begin{array}{c}\text { VOX } \\
2019\end{array}$} \\
\hline & & 2016 & 2019 & 2016 & 2019 & 2016 & 2019 & 2016 & 2019 & \\
\hline $\begin{array}{l}\text { Factor } \\
\text { Aversión }\end{array}$ & \multirow{2}{*}{$\begin{array}{l}\text { Resentimiento } \\
\text { Amargura } \\
\text { Desprecio } \\
\text { Odio } \\
\text { Asco }\end{array}$} & 88,59 & 82,02 & 77,36 & 82,92 & 89,68 & 69,43 & 79,93 & - & 55,87 \\
\hline $\begin{array}{l}\text { Alfa de } \\
\text { Cronbach }\end{array}$ & & 0,851 & 0,908 & 0,902 & 0,818 & 0,959 & 0,833 & 0,907 & 0,833 & 0,146 \\
\hline
\end{tabular}

Fuente: elaboración propia a partir de datos de los estudios EPEGE. Emociones y comportamiento electoral 2016 y EPEGE nov.19 del EIP-USC.

Tabla 7. Análisis factorial y análisis de fiabilidad de los componentes emocionales hacia los líderes políticos 2016-2019

\begin{tabular}{|c|c|c|c|c|c|c|c|c|c|c|}
\hline & & \multicolumn{2}{|c|}{ Líder PP } & \multicolumn{2}{|c|}{ Líder PSOE } & \multicolumn{2}{|c|}{$\begin{array}{c}\text { Líder } \\
\text { PODEMOS }\end{array}$} & \multicolumn{2}{|c|}{ Líder Cs } & \multirow{2}{*}{$\begin{array}{l}\text { Líder } \\
\text { VOX } \\
2019\end{array}$} \\
\hline & & 2016 & 2019 & 2016 & 2019 & 2016 & 2019 & 2016 & 2019 & \\
\hline $\begin{array}{l}\text { Factor } \\
\text { Entusiasmo }\end{array}$ & \multirow{2}{*}{$\begin{array}{l}\text { Esperanza } \\
\text { Entusiasmo } \\
\text { Orgullo } \\
\text { Tranquilidad }\end{array}$} & 75,77 & 78,81 & 71,39 & 68,70 & 68,06 & 69,98 & 75,69 & 66,12 & 69,54 \\
\hline $\begin{array}{l}\text { Alfa de } \\
\text { Cronbach }\end{array}$ & & 8,892 & 0,910 & 0,862 & 0,847 & 0,826 & 0,855 & 0,891 & 0,829 & 0,852 \\
\hline $\begin{array}{l}\text { Factor } \\
\text { Ansiedad }\end{array}$ & \multirow{2}{*}{$\begin{array}{l}\text { Miedo } \\
\text { Preocupación } \\
\text { Enfado } \\
\text { Ansiedad }\end{array}$} & 61,89 & 64,93 & 74,37 & 58,71 & 75,91 & 75,92 & 72,31 & 66,29 & 63,90 \\
\hline $\begin{array}{l}\text { Alfa de } \\
\text { Cronbach }\end{array}$ & & 0,778 & 0,802 & 0,884 & 0,752 & 0,890 & 0,893 & 0,867 & 0,795 & 0,791 \\
\hline $\begin{array}{l}\text { Factor } \\
\text { Aversión }\end{array}$ & \multirow{2}{*}{$\begin{array}{l}\text { Resentimiento } \\
\text { Amargura } \\
\text { Desprecio } \\
\text { Odio } \\
\text { Asco }\end{array}$} & 71,98 & 92,88 & 92,39 & 86,11 & 94,94 & 73,70 & 74,26 & 85,24 & 78,35 \\
\hline $\begin{array}{l}\text { Alfa de } \\
\text { Cronbach }\end{array}$ & & 0,868 & 0,975 & 0,970 & 0,957 & 0,980 & 0,882 & 0,870 & 0,954 & 0,882 \\
\hline
\end{tabular}

Fuente: elaboración propia a partir de datos de los estudios EPEGE. Emociones y comportamiento electoral 2016 y EPEGE nov.19 del EIP-USC.

A grandes rasgos, y en lo que al análisis factorial de las emociones hacia los partidos políticos se refiere, es importante señalar la existencia de un mayor nivel de explicación general para el componente aversión en los dos momentos y para el conjunto de las formaciones, con valores de varianza explicada superiores al $77 \%$ (salvo VOX y PODEMOS en 2019), llegando en algunos casos hasta el 89,68\% de Cs en 2016. Los dos supuestos en los que no se ha incluido 
el valor de varianza explicada (componente de ansiedad hacia Podemos y componente aversión hacia Cs, ambos en 2019) se debe en el primer caso al hecho de que la agrupación no se produciría en un único factor, sino en dos, y en el segundo, a un problema con el número de casos de una de las variables que interviene en la agrupación.

Respecto a los niveles de fiabilidad, los valores obtenidos para el conjunto de la escala con el estadístico Alfa de Cronbach son excelentes o buenos en la totalidad de los casos si atendemos a los valores de referencia (George y Mallery, 1995) ${ }^{19}$, salvo en el caso del componente aversión hacia VOX. En este sentido es importante señalar que se produce un problema en esta formación respecto a la variable desprecio. La eliminación de la construcción de este factor permitiría mejorar notablemente tanto los niveles de varianza explicada como el valor de fiabilidad global de la escala.

En la tabla 7 se muestra el análisis para los componentes emocionales hacia los líderes. No observamos ninguna excepción, como las mencionadas anteriormente, y todos los componentes, independientemente del año y del líder, presentan elevados niveles de explicación y de validez interna de la escala. Los valores de varianza explicada se encuentran en todos los casos por encima del $61 \%$, llegando incluso hasta el 94,94\% en el caso de la aversión hacia Pablo Iglesias en 2016. Los niveles de fiabilidad son en gran parte de los casos excelentes o buenos, y en lo tocante al componente ansiedad, aceptables. Los mayores niveles de validez interna los encontramos en los componentes de aversión y entusiasmo, por ese orden.

Para finalizar, nos gustaría resaltar que estos valores están en sintonía con los obtenidos en los trabajos basados en datos de encuesta de Marcus et al. (2006), como en los basados en método experimental (Marcus et al., 2017), y que si bien se han detectado algunas distorsiones que han sido comentadas, entendemos que entran dentro de la variabilidad que el contexto y la coyuntura pueden imprimir en cada momento, sin que ello invalide en ningún caso la agregación emocional propuesta por los autores y mantenida en nuestra propuesta.

\section{CONCLUSIONES}

Para concluir y tras haber discutido algunos de los principales hallazgos de nuestro análisis, pondremos en valor ciertas cuestiones que se desprenden

19 Si el valor del estadístico es mayor que 0.9 , el instrumento de medición es excelente; en el intervalo 0.9-0.8, es bueno; entre 0.8-0.7, es aceptable; entre 0.7-0.6, es débil; entre $0.6-0.5$, el instrumento es pobre; y si es menor que 0.5 , no es aceptable. 
del mismo, que conectan con los objetivos planteados y que nos llevan a pensar en nuevos planteamientos de cara a futuras investigaciones que amplíen y mejoren las líneas aquí esbozadas.

En primer lugar, los valores de presencia emocional tanto hacia partidos como líderes son, en líneas generales, similares en ambos procesos electorales en lo que a su aglutinación en componentes emocionales se refiere, y eso implica que aquellas emociones más negativas, que definen el componente aversión, estarían menos presentes que las que definen los componentes entusiasmo o ansiedad. El factor singular, sin embargo, se presenta con VOX, que de manera agregada presenta mayores niveles de presencia para el componente ansiedad, lo cual incide en la singularidad de los componentes emocionales de los partidos de extrema derecha.

Se observa, además, una replicación en cuanto a las emociones que mayor y menor presencia muestran en los tándems partido-líder, aunque porcentualmente mayor en estos últimos (apartado 4.1). La pareja — partido, líder- que mayor variabilidad muestra en ambos años es la de Podemos-Iglesias, y en menor medida Cs-Rivera, frente a la mayor estabilidad emocional en las formaciones tradicionales. Lo cual nos lleva a plantear la idea de que los partidos más asentados tienen más estabilizadas las emociones de los ciudadanos respecto a ellos, mientras los nuevos partidos presentan una mayor oscilación emocional.

En el caso del liderazgo del PP, dado el cambio que se produce entre los dos momentos analizados y a la vista de los resultados, cabría realizar la reflexión sobre las emociones expresadas hacia un liderazgo saliente y hacia un liderazgo en ciernes, aún no asentado y que quizás no termina de convencer, dados los porcentajes de emociones positivas que provoca, y teniendo en cuenta que en gran medida estas suelen estar sustentadas en aquellos votantes más identificados con el partido.

En segundo lugar, los valores de intensidad emocional nos permiten concluir, en líneas generales y teniendo en cuenta las diferencias descritas en el apartado 4.2., que todos los partidos presentan para las trece emociones mayores niveles de intensidad en el año 2019 que en 2016, lo que sin duda podría obedecer a un mayor clima de tensión en la competición política. Es también interesante reflejar que, si bien en el caso de las formaciones tradicionales no se produce un patrón de intensidad emocional mayor o menor asociada a una emoción concreta entre ambos momentos, sí se produce en el caso de las nuevas formaciones, Podemos y Ciudadanos, siendo una emoción negativa como el odio la que mayor intensidad muestra. Este patrón se replica en las intensidades hacia algunos liderazgos, como es el caso de Pablo Iglesias y Albert Rivera, y otros como Pedro Sánchez muestran un patrón diferenciado. Entendemos que estas cuestiones nos permitirían hablar de liderazgos superpuestos, en el primer caso, y liderazgos complementarios en el segundo en 
lo que a la intensidad emocional se refiere. Paradigmático es el caso de Santiago Abascal, puesto que es el líder que presenta mayores niveles de intensidad respecto al resto y a su propia formación, compartiendo con esta un patrón: las mayores intensidades se recogen en emociones de espectro negativo o muy negativo y las más bajas en espectro positivo. Sin duda, este planteamiento entronca con hallazgos de trabajos previos respecto al papel del líder en los partidos de extrema derecha.

En términos agregados, señalar dos cuestiones de interés: la primera, los mayores niveles de intensidad de los partidos frente a los líderes para el componente aversión en todos los supuestos tanto para el año 2016 como 2019; y en segundo lugar, la mayor intensidad del componente entusiasmo en los partidos frente a los líderes en el caso de las nuevas formaciones o de los nuevos líderes. Esto nos permitiría afirmar que en aquellas situaciones de incertidumbre sería la formación, muy posiblemente a través de los identificados, la que estaría sosteniendo su continuidad.

En tercer lugar, y en lo que a la persistencia emocional se refiere, señalar de nuevo los elevados porcentajes observados tanto en el conjunto de partidos como de líderes analizados, especialmente en el año 2016 (apartado 4.3). Otra cuestión de gran valor es la evidencia de una mayor persistencia de las emociones negativas y muy negativas frente a las emociones positivas, con la única excepción de VOX y especialmente de su líder, Santiago Abascal, en el que los mayores niveles de persistencia se expresan en emociones positivas como el orgullo (de todos modos, la persistencia aquí está ligada a límites de temporalidad más cortos que en los otros partidos).

En cuarto lugar, se confirma la validez interna de las escalas emocionales utilizadas para cada componente, con elevados niveles de fiabilidad, tanto en el caso de los partidos como de los líderes. Así mismo, los niveles de capacidad explicativa de los factores construidos en torno a los tres componentes son notables tanto en el caso de formaciones como de líderes, si bien los porcentajes son superiores en el caso de estos últimos. Aun así, algunas excepciones observadas y ya comentadas en el apartado 4.4, especialmente en lo que al partido de extrema derecha y su líder se refiere, permiten pensar en un análisis específico de cara al futuro y quizás en la consideración individual de algunas emociones concretas.

En conclusión, este trabajo ha permitido confirmar la solidez del objetivo inicial, la importancia y riqueza del componente emocional en el análisis de los partidos políticos y sus líderes, la riqueza interpretativa que brinda la consideración tridimensional de dicho factor (presencia, intensidad y persistencia) y la capacidad de compactación interna que las emociones, en las que subyacen los mismos afectos, tienen para la construcción de componentes emocionales. 


\section{Bibliografía}

Abelson, R., Kinder, D., Peters M. y Fiske, S. (1982). Affective and Semantic Components in Political Person Perception. Journal of Personality and Social Psychology, 42, 619-630. Disponible en: https://doi.org/10.1111/pops.12487.

Arias Maldonado, M. (2016). La democracia sentimental. Política y emociones en el siglo XXI. Madrid: Página Indómita.

Bodei, R. (2003). Geometria delle Passioni. Paura, Speranza e Felicità: Filosofía e Uso Político. Milan: Feltrinelli.

Bradley, M. y Lang, P. (1994). Measuring Emotion: The Self-assessment Manikin and the Semantic Differential. Journal of Behavior Therapy and Experimental Psychiatry, 25 (1), 49-59. Disponible en: https://doi.org/10.1016/0005-7916(94)90063-9.

Brady, B., Wills J., Jost J., Tucker, J. y Van Bavel, J. (2017). Emotion Shapes the Diffusion of Moralized Content in Social Networks. Proceedings of the National Academy of Sciences, 114 (28), 7313-7318. Disponible en: https://doi.org/10.1073/pnas.1618923114.

Conover, P. y Feldman, S. (1986). Emotional Reactions to the Economy: I'm Mad as Hell and I'm not Going to Take it anymore. American Journal Political Science, 30 (1), 50-78. Disponible en: https://doi.org/10.2307/2111294.

Cuik, D., Troy, A. y Jones, M. (2015). Measuring Emotion: Self-Reports vs. Physiological Indicators. SSRNElectric Journal. Disponible en: https://doi.org/10.2139/ssrn.2595359.

Damasio, A. (1994). Descartes' Error: Emotion, Reason and the Human Brain. New York: G. P. Putnam's Sons.

- (2003). Looking for Spinoza: Joy, Sorrow and the Feeling Brain. New York: Houghton Mifflin Harcourt.

Dixon, T. (2003). From Passions to Emotions. The Creation of a Secular Psychological Category. Cambridge: Cambridge University Press.

Elster, J. (1999a). Alchemies of the Mind. Cambridge: Cambridge University Press. Disponible en: https://doi.org/10.1017/CBO9781139173308.

— (1999b). Strong Feelings. Cambridge: Massachusetts Institute of Technology. Disponible en: https://doi.org/10.7551/mitpress/6498.001.0001.

George, D. y Mallery, P. (1995). SPSS/PC + Step by: A Simple Guide and Reference. Belmont (CA): Wadsworth Publishing Company.

Hasell, A. y Weeks, B. (2016). Partisan Provocation: The Role of Partisan News Use and Emotional Responses in Political Information Sharing in Social Media. Human Communication Research, 42 (4), 641-661. Disponible en: https://doi.org/10.1111/hcre.12092.

Hogget, P. (2010). Politics, Identity and Emotion. London: Taylor and Francis Ltd. y Thompson, S. (2012). Politics and the Emotions. Continuum.

Jaráiz E., Lagares, N. y Pereira, M. (2020a). Emociones y decisión de voto. Los componentes de voto en las elecciones generales de 2016 en España. Revista Española de Investigaciones Sociológicas, 170, 115-136. Disponible en: http://dx.doi.org/10.5477/cis/reis.170.115.

Jaráiz, E., López-López, P. y Bastos, M. (2020b). Economía, política social y Twitter: análisis de las emociones negativas en cuatro elecciones presidenciales latinoamericanas a través del LIWC. Revista Ibérica de Sistemas e Tecnologias de Informaçāo, E26, 270-272. 
Jaráiz, E., Pereira, M. y Rivera, R. (2020c). Análisis de emociones originadas por las publicaciones Twitter de los candidatos en las elecciones generales de Bolivia y Espańa en 2019. Journal of Iberian and Latin American Research, 26 (3), 1-18.

Jasper, J. (2012). Las emociones y los movimientos sociales: veinte ańos de teoría e investigación. Revista Latinoamericana de Estudios sobre Cuerpos, Emociones y Sociedad, 4 (10), 46-66.

Lin, R. y Utz, S. (2015). The Emotional Responses of Browsing Facebook: Happiness, Envy, and the Role of Tie Strength. Computers in Human Behavior, 52, 29-38. Disponible en: https://doi.org/10.1016/j.chb.2015.04.064.

López-López, P., Oñate, P. y Rocha, A. (2020). Social Media Mining, Debate and Feelings: Digital Public Opinion's Reaction in Five Presidential Elections in Latin America. Cluster Computing, 23, 1-12. Disponible en: https://doi.org/10.1007/s10586-020-03072-8.

Máiz, R. (2010). La hazaña de la razón: la exclusión fundacional de las emociones en la teoría política moderna. Revista de Estudios Políticos, 149, 11-45.

- (2011). The Political Mind and its Other. Rethinking the Non-place of Passions in Modern Political Theory. En M. Engelken-Jorge, P. Ibarra y C. Moreno (ed.). Politics and Emotion (pp. 29-70). VS Verlag für Sozialwissenschaften. Disponible en: https:// doi.org/10.1007/978-3-531-93201-9_2.

Marcus, G. (2000). Emotions in Politics. Annual Review of Political Science, 3, 221-250. Disponible en: https://doi.org/10.1146/annurev.polisci.3.1.221.

(2002). Sentimental Citizen: Emotion in Democratic Politics. University Park PA: Pennsylvania State University Press.

— y MacKuen, M. (1993). Anxiety, Enthusiasm and the Vote: the Emotional Underpinnings of Learning and Involvement during Presidential Campaigns. American Political Science Review, 87 (3), 688-701. Disponible en: https://doi.org/10.2307/2938743.

— , MacKuen, M., Wolak, J. y Keele, L. (2006). The Measure and Mismeasure of Emotion. En D. Redlawsk (ed.). Feeling Politics: Emotion in Political Information Processing (pp. 31-46). New York: Palgrave Macmillan. Disponible en: https://doi. org/10.1057/9781403983114_3.

— , Neuman, R. y Mackuen, M. (2000). Affective Intelligence and Political Judgement. Chicago: Chicago University Press.

— , E., Neuman, R. W. y MacKuen, M. B. (2017). Measuring Emotional Response: Comparing Alternative Approaches to Measurement. Journal of Political Science Research and Methods, 5 (4), 733-754. Disponible en: https://doi.org/10.1017/psrm.2015.65.

— , Sullivan, J., Theiss-Morse, E. y Stevens, D. (2005). The Emotional Foundation of Political Cognition: the Impact of Extrinsic Anxiety on the Formation of Political Tolerance Judgments. Political Psychology, 26 (6), 949-963. Disponible en: https://doi.org/10.1111/ j.1467-9221.2005.00452.x.

— , Valentino, N., Vasilopoulos, P. y Foucault, M. (2019). Applying the Theory of Affective Intelligence to Support for Authoritarian Policies and Parties. Advances in Political Psychology, 40 (1), 109-139. Disponible en: https://doi.org/10.1111/pops.12571.

Masters, R. (2001). Cognitive Neuroscience, Emotion, and Leadership. En J. Kuklinski. Citizens and Politics. Perspectives from Political Psychology (pp. 68-102). Cambridge: Cambridge University Press. Disponible en: https://doi.org/10.1017/CBO9780511896941.005. 
- y Sullivan, D. (1989). Nonverbal Displays and Political Leadership in France and the United States. Political Behavior, 11 (2), 123-156. Disponible en: https://doi. org/10.1007/BF00992491.

Neuman, W., Marcus, G., Grigler, A. y Mackuen, M. (2007). The Affect Effect. Dynamics of Emotion in Political Thinking and Behavior. Chicago: Chicago University Press. Disponible en: https://doi.org/10.7208/chicago/9780226574431.001.0001.

Neuman, W., Marcus, G. y Mackuen, M. (2018). Hardwired for News: Affective Intelligence and Political Attention. Journal of Broadcasting and Electronic Media, 62 (4), 614-635. Disponible en: https://doi.org/10.1080/08838151.2018.1523169.

Nussbaum, M. (1994). The Therapy of Desire. Princeton: Princeton University Press.

- (2001). Upheavals of Thought: the Intelligence of Emotions. Cambridge: Cambridge University Press. Disponible en: https://doi.org/10.1017/CBO9780511840715.

— (2013). Political Emotions: Why Love Matters for Justice. Cambridge, Massachusetts: The Belknap Press.

Parga, G. y Pereira, M. (2019). La construcción emocional de la identificación partidista. Paper presentado en el XIV Congreso de Ciencia Política y de la Administración, Salamanca.

Plutchik, R. (2002). Emotions and Life: Perspectives from Psychology, Biology and Evolution. American Psychological Association.

Ragsdale, L. (1991). Strong Feelings: Emotional Responses to Presidents. Political Behavior, 13, 33-65. Disponible en: https://doi.org/10.1007/BF00996998.

Redlawsk, D. P. (2006). Feeling Politics. Emotion in Political Information Processing. New York: Palgrave Macmillan. Disponible en: https://doi.org/10.1057/9781403983114.

Rico, G., Guinjoan, M. y Anduiza, E. (2017). The Emotional Underpinnings of populism: How Anger and Fear Affect Populist Attitudes. Swiss Political Science Review, 23 (4), 444-461. Disponible en: https://doi.org/10.1111/spsr.12261.

Russell, J. A. (1980). A circumplex model of affect. Journal of Personality and Social Psychology, 39 (6), 1161-1178. Disponible en: https://doi.org/10.1037/h0077714.

Solomon, R. (1993). The Passions. Emotions and the Meaning of Life. Indianapolis: Hacket.

Stieglitz, S. y Dang-Xuan, L. (2012). Political Communication and Influence through Microblogging. An Empirical Analysis of Sentiment in Twitter Messages and Retweet Behavior. En 2012 45th Hawaii International Conference on System Sciences (pp. 3500-3509). Disponible en: https://doi.org/10.1109/HICSS.2012.476.

Sullivan, D. y Masters, R. (1988). Happy Warriors': Leaders Facial Display, Viewers Emotions, and Political Support. American Journal of Political Science, 32 (2), 345-368. Disponible en: https://doi.org/10.2307/2111127.

Toubiana, M. y Zietsma, Ch. (2017). The Message is on the Wall? Emotions, Social Media and the Dynamics of Institutional Complexity. Academy of Management Journal, 60 (3), 922-953. Disponible en: https://doi.org/10.5465/amj.2014.0208.

Vasilopoulos, P., Marcus, G., Valentino, N. y Foucault, M. (2019). Fear, Anger, and Voting for the Far Right: Evidence from the November 13, 2015 Paris Terror Attacks. Political Psychology, 40 (4), 679-704. Disponible en: https://doi.org/10.1111/pops.12513.

Watson, D. (1997). Measurement and Mismeasurement of Mood: Recurrent and Emergent Issues. Journal of Personality Assessment, 68 (2), 267-296. Disponible en: https://doi. org/10.1207/s15327752jpa6802_4. 
- y Tellegen, A. (1999). Issues in the Dimensional Structure of Affect: Effects of Descriptors, Measurement Error, and Response Formats: Comment on Russell and Carroll. Psychological Bulletin, 125 (5), 601-610. Disponible en: https://doi.org/10.1037/00332909.125.5.601.

Westen, D. (2007). The Political Brain: The Role of Emotion in Deciding the Fate of the Nation. New York: Public Affairs.

Yates, H. (2016). The Politics of Emotions, Candidates and Choices. Nueva York: Palgrave Macmillan. Disponible en: https://doi.org/10.1057/978-1-137-51527-8. 